\title{
Mechanism of life-long maintenance of neuron identity despite molecular fluctuations
}

\author{
Joleen J. H. Traets ${ }^{1}$, Servaas N. van der Burght ${ }^{2}$, Gert Jansen ${ }^{2}$, Jeroen S. van Zon ${ }^{1 *}$
}

${ }^{1}$ AMOLF, Science Park 104, 1098 XG Amsterdam, the Netherlands.

${ }^{2}$ Department of Cell Biology, Erasmus University Medical Centre, Dr. Molewaterplein 40, 3015 GD, Rotterdam, the Netherlands.

"lead contact: j.v.zon@amolf.nl

\section{Summary}

Cell fate is maintained over long timescales, yet molecular fluctuations can lead to spontaneous loss of this differentiated state. We uncovered a mechanism that explains lifelong maintenance of ASE neuron fate in C. elegans by the terminal selector transcription factor $\mathrm{CHE}-1$. Fluctuations in $\mathrm{CHE}-1$ level are buffered by the reservoir of $\mathrm{CHE}-1$ bound at its target promoters, which ensure continued che-1 expression by preferentially binding the che-1 promoter. We validated this mechanism by showing that che-1 expression was resilient to induced transient $\mathrm{CHE}-1$ depletion, while both expression of $\mathrm{CHE}-1$ targets and ASE function were lost. We identified a 130 bp che-1 promoter fragment responsible for this resilience, with deletion of a homeodomain binding site in this fragment causing stochastic loss of ASE identity long after its determination. Because network architectures that support this mechanism are highly conserved in cell differentiation, it may explain stable cell fate maintenance in many systems.

\section{Keywords}

Neuronal cell fate, bistability, gene regulatory network, stochastic gene expression, molecular fluctuations, homeodomain proteins, chemotaxis, C. elegans, terminal selector 


\section{Introduction}

In most animal tissues, terminally differentiated cells are renewed on timescales of days to months (Leblond and Walker 1956), yet the nervous system is unique, as most neurons hardly renew at all (Ming and Song 2005). Many mature neuron subtypes exist that differ in the expression of hundreds of subtype-specific genes (Deneris and Hobert 2014). How neuronal cells maintain this terminally differentiated state over such long timescales decades, in the case of humans - is not understood.

Recent studies have found that differentiation of neuron subtypes is often controlled by only 1 or 2 different transcription factors, called "terminal selectors" (Hobert 2016). These act through a conserved network motif called a single-input module (Alon 2007): they bind to specific cis-regulatory control elements to induce both their own expression and that of the downstream target genes that define the neuronal subtype (Fig. 1A). Such terminal selector networks have been found to underlie differentiation of many neuron types in the nematode Caenorhabditis elegans (Deneris and Hobert 2014; Hobert 2016), photoreceptor subtypes in Drosophila (Hsiao et al. 2013) and dopaminergic neurons in mice (Ninkovic et al. 2010), indicating that they form an evolutionary conserved principle for neuron subtype determination.

Terminal selectors positively regulate their own expression, raising the possibility that they act as bistable genetic switches. Such switches are wide-spread in biology (Ferrell 2002; Alon 2007) and are often seen as an attractive mechanism to explain cell fate determination. In this hypothesis, at the time of determination, transient signals induce expression of terminal selectors, which then maintain their own expression, and that of all target genes, by autoregulation in the subsequent absence of these signals (Fig. 1B) (Hobert 2008). However, a key weakness of bistable switches is that they remain reversible at all times, with a transient decrease in terminal selector levels potentially sufficient to lose terminal selector expression and, presumably, cell fate (Fig. 1B). Indeed, bistable genetic switches often suffer from stochastic transitions between their different states due to molecular noise, i.e. random fluctuations in the levels of their core components (Ozbudak et al. 2004; Suel et al. 2006; Acar $t$ al. 2005). Hence, it is often assumed that stable cell fate maintenance must require additional feedback mechanisms, such as histone or chromatin modifications (Orlando 2003; Ringrose and Paro 2007), that lock-in cell fate in an essentially irreversible manner.

Here, we studied long-term maintenance of neuron subtype in the salt-sensing ASE neurons of the nematode C. elegans (Fig. 1A). ASE subtype is controlled by the terminal selector 
CHE-1, a transcription factor whose expression is transiently induced by the nuclear hormone receptor NHR-67 at the time of determination (Sarin et al. 2009). CHE-1 induces the expression of 500-1000 ASE-specific target genes, such as chemosensory receptors, ion-channels, and neuropeptides, by binding ASE motifs within their promoters (Etchberger et al. 2007). Its continued presence is required for expression of target genes after subtype determination (Etchberger et al. 2009). CHE-1 also upregulates its own expression. This positive feedback loop is necessary for sustaining che-1 expression and ASE cell fate directly after cell determination (Etchberger et al. 2007; Leyva-Diaz and Hobert 2019). Yet, it is unknown whether this positive feedback loop is sufficient for long-term maintenance of ASE fate. The impact of molecular noise, such as variability in CHE-1 protein copy number, on ASE fate maintenance has not been studied. Overall, it is an open question whether a reversible, bistable switch based on positive CHE-1 autoregulation would be sufficiently stable to maintain ASE fate for the animal's lifetime, or if additional mechanisms are necessary to ensure that, once ASE fate is determined, che-1 expression becomes independent of $\mathrm{CHE}-1$ itself and can no longer spontaneously switch off.

We show that sufficiently long, transiently induced depletion of CHE-1 caused permanent loss of ASE fate, indicating that it is controlled by a switch that remains reversible long after specification. This raises the question how the switch is protected against molecular noise, which could cause it to spontaneously lose ASE fate. Combining experimental measurements of the key parameters that control the magnitude of noise, i.e. the copy numbers and lifetimes of che-1 mRNA and protein, with stochastic models of the che-1 genetic network, revealed a novel mechanism, "target reservoir buffering", that dramatically increased switch stability. This stability resulted from the presence of a reservoir of $\mathrm{CHE}-1$ protein bound at the promoters of its target genes, coupled with preferential binding of CHE1 to the che-1 promoter compared to the promoters of its other targets. This led to exceedingly stable ON states (high che-1 expression), with spontaneous transitions to the OFF state (no che-1 expression) observed at rates of $<10^{-3} /$ year. Consistent with this mechanism, we found that upon induced $\mathrm{CHE}-1$ depletion in vivo, che-1 mRNA expression remained present, even when expression of other target genes vanished together with the animal's ability to respond to salt. This allowed full recovery of $\mathrm{CHE}-1$ protein levels, target gene expression and chemosensation if induced CHE-1 depletion was sufficiently short. We found a 130 bp promoter region surrounding the che-1 ASE motif responsible for this resilience of che-1 expression to $\mathrm{CHE}-1$ depletion. This region contained a homeodomain protein binding site that, when mutated, caused stochastic loss of ASE fate well after ASE specification, indicating a strong decrease in stability of the $\mathrm{ON}$ state. This shows a new role 
bioRxiv preprint doi: https://doi.org/10.1101/2020.04.22.054536; this version posted April 22, 2020. The copyright holder for this preprint (which was not certified by peer review) is the author/funder. All rights reserved. No reuse allowed without permission.

for homeodomain proteins in maintaining and stabilizing ASE fate, likely by recruiting CHE-1 preferentially to its own promoter. 


\section{Results}

\section{Loss of ASE neuron fate upon transient CHE-1 depletion}

To test whether positive autoregulation of che-1 expression is necessary for ASE fate maintenance, we depleted CHE-1 protein levels in ASE neurons in vivo, using the auxin inducible degradation system (Zhang et al. 2015). che-1::GFP::AID animals were exposed to $1 \mathrm{mM}$ auxin to induce CHE-1 depletion (Fig. 1C). CHE-1::GFP was strongly reduced after $\sim 1.5 \mathrm{hr}$, and undetectable after $\sim 4 \mathrm{hrs}$ exposure to auxin (Fig. 1D). To examine how CHE-1 depletion impacted ASE function, we used a quadrant chemotaxis assay to quantify the chemotaxis response of CHE-1 depleted animals (Wicks et al. 2000; Jansen et al. 2002). In this assay, animals choose between two agar quadrants with $\mathrm{NaCl}$ and two without (Fig. S1A). che-1::GFP::AID animals exposed to auxin for $24 \mathrm{hrs}$ showed reduced chemotaxis to $\mathrm{NaCl}(\mathrm{P}<0.001)$ (Fig. 1E, S1C). This agrees with previous results showing that permanent inhibition of che-1 expression by RNAi in larvae resulted in reduced expression of $\mathrm{CHE}-1$ target genes (Etchberger et al. 2009). However, these results leave open the possibility that, after initial ASE subtype determination, che-1 expression is maintained independently of $\mathrm{CHE}-1$ in an irreversible manner.

In contrast, if che-1 expression is bistable and reversible, then a sufficiently long period of transient $\mathrm{CHE}-1$ depletion should result in permanent loss of che-1 expression (Fig. 1B). To test this, we exposed animals to auxin for increasing time intervals (Fig. 1C), and analysed CHE-1::GFP expression and $\mathrm{NaCl}$ chemotaxis after 24 (Fig. 1D, S1B,C) or 48 hrs (Fig. $\mathbf{1 E}, \mathbf{F})$ recovery on plates without auxin. In animals exposed to auxin for $24 \mathrm{hrs}$, both CHE$1::$ GFP expression and $\mathrm{NaCl}$ chemotaxis returned to wild-type levels after 24 hrs recovery. However, after 48 hrs auxin exposure, CHE-1::GFP did not return in 11/41 animals (Fig. 1F, S1B). This fraction further decreased with the duration of auxin exposure, with 0/9 animals recovering CHE-1::GFP expression after $120 \mathrm{hrs}$ on auxin. Similarly, the chemotaxis response did not recover in animals exposed to auxin for 96 hrs or longer (Fig. 1E, S1C; Table S1), suggesting that the absence of CHE-1::GFP resulted in loss of ASE subtype identity. Overall, these observations indicated that $\mathrm{CHE}-1$ controls its expression, and ASE fate, as a bistable switch that, even though it can withstand strong decreases in CHE-1 level for a limited time, remains fully reversible long after its induction. 


\section{Copy number and lifetime of che-1 mRNA and protein}

The observed reversibility of the ON state raises the question how spontaneous transitions to the OFF state, due to random fluctuations in CHE-1 level, are prevented under normal conditions. Theoretical studies of genetic switches suggest that the probability of such transitions decreases with increasing average copy number and lifetime of the transcription factors involved (Warren and ten Wolde 2005; Walczak et al. 2005; Mehta et al. 2008). Therefore, we determined copy numbers and lifetimes of che-1 mRNA and protein.

First, we measured the absolute number of che-1 mRNA and protein molecules in ASE neurons. We used single-molecule FISH (smFISH) (Ji and van Oudenaarden 2012) to count individual che-1 mRNA molecules. As a benchmark, we also measured mRNA levels of the putative $\mathrm{NaCl}$ receptors gcy-14 and gcy-22, and the ion channel subunits del-2 and tax-2, which are CHE-1 target genes with $>1$ ASE motif in their promoter region (Ortiz et al. 2009; Coburn and Bargmann 1996). In embryos, we found that che-1 mRNA levels peaked at $26 \pm 6$ mRNAs/cell during the bean stage, i.e. the time of ASE neuron determination (Fig. 2A,B), and fell to $6 \pm 3 \mathrm{mRNAs} / \mathrm{cell}$ from the comma stage onwards. In contrast, the CHE-1 target gene gcy-22 showed a steady increase in mRNA levels during development and surpassed che-1 mRNA expression after the 1.5-fold stage. In larvae, we also found low che-1 mRNA levels, with $5 \pm 2$ mRNAs in the left (ASEL) and $7 \pm 3$ mRNAs in the right ASE neuron (ASER). che-1 expression was low compared to the panel of $\mathrm{CHE}-1$ target genes examined (Fig. 2C,D), with del-2, gcy-14 and gcy-22 expressed at significantly higher levels. Moreover, che-1 mRNA levels remained low during all four larval stages, L1-L4, with slightly higher mRNA levels in the ASER, compared to the ASEL neuron (Fig. 2E). For this entire period, the target genes with the highest expression, gcy-14 and gcy-22, remained expressed well above che-1 levels. Overall, these results show that che-1 expression is biphasic: an initiation phase with high che-1 expression level when ASE fate is induced in the embryo, followed by a maintenance phase with che-1 expressed at a low level where molecular copy number fluctuations likely have significant impact.

We also estimated the absolute $\mathrm{CHE}-1$ protein copy number in the ASE neurons. We immersed endogenously tagged che-1::GFP animals (Leyva-Diaz and Hobert 2019) in eGFP, and calculated the CHE-1 protein number by comparing the CHE-1::GFP fluorescence inside the ASE neurons with the ambient fluorescence of eGFP (Fig. 2F) (Gregor et al. 2007). For all stages of post-embryonic development, we found an average

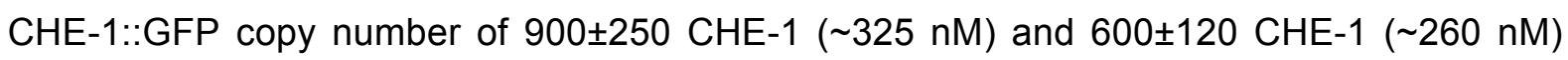


molecules/cell for the ASER and ASEL neuron, respectively (Fig. 2F, S2A), with slightly lower copy numbers observed in the embryo (Fig. S2B). Overall, the observed range of CHE-1::GFP protein levels, 500-1400 molecules/cell, is comparable to the number of CHE-1 binding sites, 500-1000, in the promoters of CHE-1 target genes, as estimated by the number of ASE motifs detected previously (Etchberger et al. 2007) and by our analysis (STAR Methods), indicating that $\mathrm{CHE}-1$ targets compete for a limited pool of $\mathrm{CHE}-1$ protein.

Next, we measured che-1 mRNA and protein lifetimes. To determine che-1 mRNA lifetimes in ASE neurons, we transiently overexpressed che-1 mRNA, using a hsp16.41p::che-1 heat shock inducible construct (Patel and Hobert 2017). By exposing animals to $37^{\circ} \mathrm{C}$ for $\sim 10 \mathrm{~min}$, we raised che-1 mRNA levels in the ASE neurons 5 -fold, to $24 \pm 4 \mathrm{mRNAs} / \mathrm{cell} \sim 10 \mathrm{~min}$ after heat shock (Fig. 3A,B). Next, we shifted animals to $20^{\circ} \mathrm{C}$ to return che-1 expression to its pre-induction level and fixed animals at $\sim 17$ min intervals to quantify che-1 mRNA levels using smFISH. We found that che-1 mRNA levels returned to pre-induction values after $\sim 60 \mathrm{~min}$. By fitting the measured che-1 mRNA levels to an exponential function, we obtained a che-1 mRNA half-life of $17 \pm 4$ min. To measure CHE-1 protein lifetime, we used Fluorescence Recovery after Photobleaching (FRAP) in CHE$1::$ GFP animals. CHE-1::GFP was bleached to approximately $\sim 20 \%$ of the original fluorescence level and we measured the recovery of the CHE-1::GFP signal over time, until it reached pre-bleaching levels, which occurred within $\sim 3 \mathrm{hrs}$ (Fig. 3C,D). To estimate CHE$1::$ GFP protein lifetime, we fitted an exponential recovery curve to the experimental data for each individual animal, resulting in an average measured half-life of $83 \pm 20 \mathrm{~min}$.

The che-1 mRNA and protein half-lives are short compared to reported mRNA halflives in eukaryotes (Fornasiero et al. 2018; Sharova et al. 2009) and average protein halflives reported in young C. elegans adults (Dhondt et al. 2017; Schwanhausser et al. 2011). Overall, these measured lifetimes show that che-1 mRNA and protein turnover is rapid compared to the 2-3 week lifespan of $C$. elegans during which ASE identity should be maintained.

\section{Stochastic simulations identify stable cell fate maintenance parameters}

The measurements of che-1 mRNA and protein copy numbers and lifetimes allowed us to perform realistic simulations of the $\mathrm{CHE}-1$ switch to estimate its stability against stochastic fluctuations. We constructed stochastic models that included production and decay of che-1 mRNA and protein molecules, binding of $\mathrm{CHE}-1$ to the promoter of che-1 and target genes, and target gene expression (Fig. 4A). We examined two bistable models that differed in 
binding of $\mathrm{CHE}-1$ to its own promoter. In the first model, we assume that $\mathrm{CHE}-1$ binds as a monomer to induce che-1 expression. This model agrees with the observation that the che-1 promoter contains only a single ASE motif (Etchberger et al. 2007), but lacks cooperativity in che-1 induction. Because cooperativity is considered important for generating bistability (Ferrell 2002), we also included a second model where che-1 induction is cooperative, by assuming that expression occurs only when two $\mathrm{CHE}-1$ molecules bind the che-1 promoter.

The two models have 8 and 9 parameters, respectively, of which experimental data fixed 6 (STAR Methods). The production and degradation rates of che-1 mRNA $\left(f_{m}, b_{m}\right)$ and protein $\left(f_{c}, b_{c}\right)$ were fully determined by the measured copy numbers and lifetimes. For the CHE-1 binding rate $\left(f_{O}\right)$ we assumed the diffusion-limited rate, i.e. the highest physically possible binding rate. Based both on previous analysis (Etchberger et al. 2007) and our own, we examined model dynamics for $N_{T}=500$ or $1000 \mathrm{CHE}-1$ target genes, i.e. either smaller or larger than the mean number of CHE-1 proteins (900 molecules/cell). The only free parameters were dissociation rates of CHE-1 from its own promoter $\left(b_{0}\right.$ or $\left.b_{1}, b_{2}\right)$ and from the other targets $\left(b_{T}\right)$. We varied these between $0.1-100 \mathrm{~s}^{-1}$, corresponding to dissociation constants of $\sim 1-10^{3} \mathrm{nM}$ and consistent with values measured for transcription factors (Gebhardt et al. 2013; Jung et al. 2018). We simulated the models using the Gillespie algorithm (Gillespie 1977). In general, our stochastic simulations showed that molecular noise was sufficiently strong to induce spontaneous transitions from the ON to the OFF state on the timescale of hours or days, indicating that the measured copy numbers and lifetimes by themselves were not sufficient to generate stability against fluctuations. However, we also identified parameter combinations for which the CHE-1 switch remained in the ON state for at least a week (Fig. 4B).

For simulations with high switch stability, brute-force Gillespie simulations were too computationally demanding to directly measure the ON state lifetime. Instead, we used Forward Flux Sampling (FFS), a computational method to efficiently sample rare transition paths between states in multi-stable systems (Allen, Frenkel, and ten Wolde 2006). Using this approach, we observed parameter combinations with very high stability, i.e. lifetimes of many years, independent of the degree of cooperativity or number of target genes (Fig. 4C, S3A,B). In general, we observed increasing lifetimes for decreasing $b_{0}$ or $b_{1}, b_{2}$, i.e. higher occupancy of the che-1 promoter by $\mathrm{CHE}-1$. Moreover, the longest lifetimes were found when $b_{0}, b_{2} \ll b_{T}$, i.e. when CHE-1 had a much higher affinity for its own promoter than for its other targets. In this regime, we observed average lifetimes of $>1 \cdot 10^{5}$ years. Note, however, that despite the long lifetimes in this regime, spontaneous transitions to the OFF state are 
rapid and occur as a random Poisson process, with a transition possible at any time, albeit with low probability. For such a Poisson process, ON state lifetimes of $>2 \cdot 10^{5}$ years are required for the probability of spontaneous loss of ASE fate during their $\sim 2$ week lifetime to be less than $10^{-6}$ (STAR Methods), the frequency of spontaneous mutations per gene per generation in C. elegans (Anderson 1995).

\section{Stability against stochastic fluctuations by preferential binding of $\mathrm{CHE}-1$ to its own promoter}

We found that high stability of the $\mathrm{ON}$ state required $b_{0}, b_{2} \ll b_{T}$, i.e. CHE- 1 binds its own promoter much more strongly than that of its other targets. An explanation for this emerged when we compared transition paths for spontaneous transitions to the OFF state, between parameter combinations that exhibited high $\left(>1 \cdot 10^{5}\right.$ years lifetime), medium $(\sim 12$ days lifetime) and low ( $\sim 5 \mathrm{hrs}$ lifetime) stability (Fig. 4D, S3C). For parameters with low stability we found that, as CHE-1 protein levels fell during spontaneous transitions to the OFF state, both the average che-1 mRNA number and the fraction of $\mathrm{CHE}-1$ target promoters occupied by $\mathrm{CHE}-1$ decreased, with very low occupancy even of the che-1 promoter itself close to the end of the transition. In contrast, for parameters with high stability, we found that che-1 promoter occupancy was high, and the che-1 promoter remained bound by $\mathrm{CHE}-1$ until the end of the transition, whereas $\mathrm{CHE}-1$ binding was lost earlier on other promoters. These results suggested that high stability arises as a result of a strong preference for $\mathrm{CHE}-1$ protein to bind to the che-1 promoter, thereby making che-1 expression insensitive to strong, stochastic decreases in $\mathrm{CHE}-1$ level.

To test this idea, we ran simulations that included a transient depletion of CHE-1, implemented by a temporary increase in the $\mathrm{CHE}-1$ protein degradation rate reducing $\mathrm{CHE}$ 1 to 100 molecules/cell (Fig. 4E). Indeed, we found that simulations with unstable switches, i.e. where $b_{0}, b_{2}>b_{T}$, were highly sensitive to such depletions, with che-1 mRNA rapidly falling to such low levels that $\mathrm{CHE}-1$ protein levels and, hence, the ON state, were not recovered when CHE-1 depletion ceased. In contrast, even though the mRNA levels of target genes fell rapidly, simulations with highly stable switches maintained normal che-1 mRNA levels for many days (Fig. 4F, S3D), allowing the system to successfully recover $\mathrm{CHE}-1$ protein levels and the $\mathrm{ON}$ state if $\mathrm{CHE}-1$ depletion was removed sufficiently rapidly.

In vivo CHE-1 depletion decreases target gene but not che-1 expression. 
These simulation results were similar to our experimental observation that CHE-1::GFP levels fully recovered even after 24-48 hours of induced CHE-1 depletion (Fig. 1C-F, S1B,C). To test whether this reflected insensitivity of che-1 expression to low CHE-1 protein levels (Fig. 4E,F), we used smFISH to compare the impact of auxin-mediated CHE$1::$ GFP::AID depletion at the mRNA levels of che-1 and other target genes, focusing on gcy22 as the most highly expressed in our panel (Fig. 5A,B). Indeed, gcy-22 mRNA levels were very low in most che-1::GFP::AID animals after 24 hrs on auxin. In striking contrast, che-1 mRNA levels were close to wild-type. After 24 hrs without auxin, gcy-22 mRNA levels had increased significantly (Fig. 5A,B), consistent with the recovery of CHE-1::GFP levels and chemotaxis to $\mathrm{NaCl}$ in these animals (Fig. 1D,E). Overall, these results were in full agreement with our model predictions but raised the question what properties of the che-1 promoter were responsible for its resilience to CHE-1 depletion.

\section{Sequences flanking the che-1 ASE motif are required for che-1 expression during CHE-1 depletion}

Previous studies identified a single ASE motif 242 bp upstream of the che-1 ATG start codon as required for autoregulation of che-1 expression (Etchberger et al. 2007; Leyva-Diaz and Hobert 2019). This ASE motif differs in 6 bp from the sequence in the gcy-22 promoter, which might explain the divergent effects of $\mathrm{CHE}-1$ depletion on che-1 and gcy-22 expression. To test this hypothesis, we used CRISPR/Cas9 in che-1::GFP::AID animals to replace the 12 bp ASE motif in the che-1 promoter with that of gcy-22, and vice versa (Fig. 6A). The resulting mutant animals showed wild-type chemotaxis to $\mathrm{NaCl}$ and exhibited che-1 and gcy-22 mRNA levels similar to wild-type (Fig. 6B,F, S4A), indicating that replacing ASE motifs did not impact ASE specification and target gene expression. Moreover, when we depleted CHE-1 protein using auxin, gcy-22 expression in (ASEche-1)::gcy-22 animals almost completely vanished (Fig. 6F), as in animals with a wild-type gcy-22 promoter. Overall, the ASE motif itself could not explain the observed differences in che-1 and gcy-22 expression. This agrees with previous results that showed a similar calculated affinity score of CHE-1 for the che-1 and gcy-22 ASE motif, despite sequence differences (Etchberger et al. 2009).

To examine if promoter regions other than the ASE motif were responsible for the differences in che-1 and gcy-22 expression, we replaced ASE motifs together with 59 bp flanks on either side (Fig. 6A). (ASE $E_{g c y-22}+$ flanks)p::che-1 animals exhibited a strong chemotaxis defect and lack of CHE-1::GFP expression in ASE neurons of larvae (Fig. 6B, 
S4B). At the pre-bean stage, (ASE $E_{g c y-22}+$ flanks)p::che-1 embryos showed high che-1 expression, 28 $\pm 7 \mathrm{mRNA} /$ cell, and CHE-1::GFP (Fig. 6C,D, S4B), indicating that che-1 expression was initiated normally during ASE determination. However, che-1 mRNA was absent in $\left(A S E_{g c y-22}+\right.$ flanks)p::che-1 embryos at later stages. This phenotype was also seen in the che-1(p679) loss of function mutant (Fig. S4C) and shows that this 130 bp che-1 promoter region is important for maintenance of che-1 expression and ASE fate. (ASE $E_{\text {che- }}$ ${ }_{1}+$ flanks)p::gcy-22 animals showed wild-type chemotaxis to $\mathrm{NaCl}$ (Fig. 6B) and gcy-22 mRNA levels (Fig. 6F). Yet, strikingly, upon CHE-1::GFP::AID depletion in (ASE che${ }_{1}+$ flanks)p::gcy-22 animals gcy-22 mRNA levels remained high (Fig. 6F), indicating that, like che-1, gcy-22 expression became resilient to CHE-1 depletion. Hence, the 130 bp che-1 promoter fragment surrounding the ASE motif is responsible for maintaining expression during CHE-1 depletion.

\section{Involvement of an Otx-related homeodomain binding site in maintaining ASE subtype}

Within the 130 bp che-1 promoter fragment required for resilient che-1 expression, we identified a high scoring Otx-related homeodomain transcription factor (HD-TF) binding site, 29 bp downstream of the che-1 ASE motif (Fig. 7A), with only 60 CHE-1 targets exhibiting a binding site of similar score within $100 \mathrm{bp}$ of their ASE motifs (Table S2). To test the function of this HD-TF binding site, we deleted it from the che-1 promoter in the che$1:: G F P:: A I D$ background. These $(\Delta H D) p:: c h e-1$ animals showed an intermediate chemotaxis defect (Fig. 7D). To examine whether this defect reflected changes in che-1 expression we scored CHE-1::GFP expression at different larval stages (Fig. 7B). CHE-1::GFP was expressed in all embryos, but was progressively lost over time, with CHE-1::GFP absent in more than half of the young adults (2/18 animals for ASEL and 9/20 for ASER), indicating a defect in maintenance of che-1 expression, not in ASE determination. We then used timelapse microscopy (Gritti et al. 2016) to monitor the dynamics of CHE-1::GFP expression directly in single $(\Delta H D) p::$ che-1 larvae (Fig. S5A). Strikingly, CHE-1::GFP expression was lost in a rapid, stochastic manner (Fig. 7E, S5A), as would be expected for spontaneous, noise-driven transitions to the OFF state. Theoretical studies showed that the rate of such transitions increases dramatically with decreasing copy number of the key transcription factors involved (Warren and ten Wolde 2005). Indeed, ( $\triangle H D) p::$ che-1 animals showed lower CHE-1::GFP fluorescence, corresponding to $190 \pm 70 \mathrm{CHE}-1$ proteins/cell (Fig. 7E, S5B) and decreased che-1 mRNA levels, with $1 \pm 1$ mRNAs/cell in L1 larvae (Fig. 7C). In addition, CHE-1::GFP expression was lost more often and earlier in development in ASEL neurons (Fig. 7B,E), which have lower average che-1 mRNA and protein copy numbers 
bioRxiv preprint doi: https://doi.org/10.1101/2020.04.22.054536; this version posted April 22, 2020. The copyright holder for this preprint (which was not certified by peer review) is the author/funder. All rights reserved. No reuse allowed without permission.

than ASER neurons (Fig. 2D,F). Altogether, these results point to homeodomain proteins, that bind the HD-TF binding site, as essential for long-term maintenance of che-1 expression and thus ASE cell fate, likely by protecting the ON state against low $\mathrm{CHE}-1$ copy number fluctuations. 


\section{Discussion}

The terminal selector gene che-1 controls subtype determination of the salt-sensing ASE neurons, by inducing the expression of hundreds of ASE-specific target genes while also inducing its own expression via autoregulation (Etchberger et al. 2007). It is an open question whether the positive autoregulation of CHE-1 is sufficient to maintain its own expression and, hence, ASE subtype, for the animal's entire lifetime, or whether additional mechanisms are necessary to lock in cell identity in an irreversible manner. Here, we show that transient depletion of CHE-1 is sufficient to permanently lose ASE function, indicating that che-1 expression forms a reversible, bistable switch. This also raised the question how, in the presence of the inherent molecular fluctuations in the cell, this switch maintains its $\mathrm{ON}$ state (che-1 expression) and prevent spontaneous transitions to the OFF state (no che-1 expression).

Theoretical studies found that the stability of bistable genetic switches against fluctuations is enhanced by increasing the copy number and lifetime of the transcription factors that form the switch (Warren and ten Wolde 2005; Walczak et al. 2005; Mehta et al. 2008). However, our simulations using in vivo measured che-1 mRNA and protein copy numbers and lifetimes showed that these values by themselves were not sufficient to generate a stable ON state. Instead, our simulations suggested a novel mechanism required to stabilize the $\mathrm{ON}$ state, which uses the reservoir of $\mathrm{CHE}-1$ proteins bound to its many target sites to buffer che-1 expression against fluctuations in CHE-1 level. Crucial to this target reservoir buffering mechanism is that $\mathrm{CHE}-1$ exhibits strong preferential binding to its own promoter compared to its other targets. In this case, when CHE-1 protein levels drop, any $\mathrm{CHE}-1$ protein that dissociates from a target gene promoter will immediately bind the che-1 promoter, if it is found unoccupied. This ensures maintenance of che-1 expression, at the expense of expression of its other targets, to bring $\mathrm{CHE}-1$ protein levels back to the normal steady state level. Our experimental observations support this mechanism. Most importantly, upon transient CHE-1 depletion in vivo, expression of the CHE-1 target gcy-22 rapidly and strongly decreased, yet che-1 expression itself was hardly affected. We show that this resilience of che-1 expression to $\mathrm{CHE}-1$ depletion could be conferred onto other target genes by introducing a $130 \mathrm{bp}$ fragment of the che-1 promoter that surrounds the ASE motif bound by CHE-1, but not the ASE motif itself.

Target reservoir buffering relies on CHE-1 being preferentially recruited to the che-1 promoter. We found evidence that homeodomain transcription factors are responsible for this. Deleting a HD-TF binding site close to the che-1 ASE motif and specific to the che-1 
promoter, caused spontaneous transitions to the OFF state, and loss of cell identity, long after ASE subtype determination. This lack of stability was accompanied by lower che-1 mRNA and protein levels, consistent with reduced recruitment of che-1 to its own promoter. We speculate that HD-TFs act by increasing the residence time of $\mathrm{CHE}-1$ at the che-1 promoter. Our simulations showed that highly stable ON states require a relatively small, 10100 fold increase in $\mathrm{CHE}-1$ residence time at the che-1 promoter compared to its other targets, within the range expected if $\mathrm{CHE}-1$ and a HD-TF interact cooperatively with a weak interaction of a few $k_{B} T$. A similar interaction was postulated for the homeodomain protein ALR-1 in mechanosensory TRN neurons, where it restricts variability in expression of the terminal selector gene mec-3, by binding close to the MEC-3 binding site on its promoter (Topalidou and Chalfie 2011). The only protein with high predicted affinity to the HD-TF binding site in the che-1 promoter, and expressed in ASE neurons, is the Otx-related homeodomain transcription factor CEH-36 (Lanjuin et al. 2003). However, a ceh-36 loss-offunction mutant (Chang et al. 2003) did not reproduce the loss of ASE identity seen upon deletion of the homeodomain binding site (data not shown), indicating a role for other ASEexpressed homeodomain proteins, potentially in combination with $\mathrm{CEH}-36$. In general, homeodomain proteins play an important role in development of the nervous system, that is conserved from C. elegans to mammals (Hobert 2010; Hench et al. 2015). Our work now extends this role also to long-term maintenance of cell fate in the face of stochastic fluctuations.

The observed resilience of che-1 expression to CHE-1 depletion is crucial for stable maintenance of ASE identity. In both our models with and without cooperativity, this is achieved by a very low threshold for inducing che-1 expression, with a single che-1 mRNA potentially sufficient to induce the ON state. Indeed, the recovery of CHE-1::GFP expression after 24-48 hrs of CHE-1 depletion demonstrates that the ON state can be recovered from very low $\mathrm{CHE}-1$ levels. However, this raises the question how stochastic, spontaneous induction of che-1 expression and ASE fate is prevented in non-ASE cells. In most cells, spontaneous induction of the $\mathrm{ON}$ state is likely prevented by chromatin remodelling (Tursun et al. 2011; Patel and Hobert 2017). However, transient che-1 expression induced long-term expression of CHE-1 targets in non-ASE head sensory neurons, suggesting that these cells are capable of inducing the ON state (Tursun et al. 2011). A potential mechanism to prevent ectopic induction of the $\mathrm{ON}$ state is provided by our observation that preferential binding of $\mathrm{CHE}-1$ to its own promoter likely depends on homeodomain proteins. Cells lacking these proteins would have difficulty inducing and maintaining che-1 expression. Consistent with this hypothesis, a large number of HD-TFs is expressed in the $C$. elegans nervous system in a highly neuron-specific manner (Hobert 2010). Our work suggests that cell-specific 
expression of co-factors of terminal selector genes might form a general mechanism to prevent spontaneous induction of these genes in the wrong cells.

While bistability in genetic networks is recognized as an important mechanism to generate cell fate switches (Ferrell 2002), long-term cell fate maintenance is often assumed to require additional feedback mechanisms, for instance through histone and chromatin modifications, that make cell fate essentially irreversible. Here, we show that bistability through an autoregulatory feedback loop alone is sufficient for life-long maintenance of neuron identity in C. elegans, despite strong stochastic molecular fluctuations in the underlying genetic network. The mechanism responsible for this, target reservoir buffering, depends crucially on the single-input module topology of the network, with a terminal selector, $\mathrm{CHE}-1$, inducing both its own expression and that of many other target genes. Single-input modules are highly prevalent network motifs for neuron subtype determination, both in C. elegans and higher organisms (Hobert and Kratsios 2019). In addition, cell differentiation in general is often controlled by a small number of master regulators that, directly or indirectly, induce both their own expression and that of many cell fate-specific target genes. Hence, we expect target reservoir buffering to play an important, general role in explaining stable long-term maintenance of cell fate in a broad array of systems.

Our work also underscores the link between biophysical parameters, such as molecular copy number and half-life, and stable cell fate maintenance. In mammals, stable maintenance of (neuronal) cell fate is even more challenging, given that the number of cells and their required lifetime is vastly larger compared to $C$. elegans. It will be important to determine if simply increasing copy number and half-life of a mammalian terminal selector is already sufficient, when coupled with target reservoir buffering, to explain the exceeding stability of cell fate in mammals. Our work now provides an experimental and theoretical framework to address this question. 


\section{Acknowledgements}

Some strains were provided by the CGC, which is funded by NIH Office of Research Infrastructure Programs (P40 OD010440), and the Mitani laboratory through the National Bio-Resource Project of the MEXT, Japan. We thank Dylan Rahe (Hobert lab) for providing the GFP-tagged che-1(ot856) allele. This work is part of the research program of the Foundation for Fundamental Research on Matter (FOM), which is financially supported by the Netherlands Organization for Scientific Research (NWO).

\section{Author contributions}

J.J.H.T, S.N.B., G.J. and J.S.Z conceived the study. J.J.H.T performed smFISH, time-lapse and (confocal) microscopy expression experiments and data analysis. S.N.B. generated strains, performed behavioural assays and data analysis. J.J.H.T and J.S.Z. performed mathematical modelling. J.J.H.T, S.N.B., G.J. and J.S.Z. wrote the manuscript.

\section{Declaration of interest}

The authors declare no competing interests 
Figure titles + legends
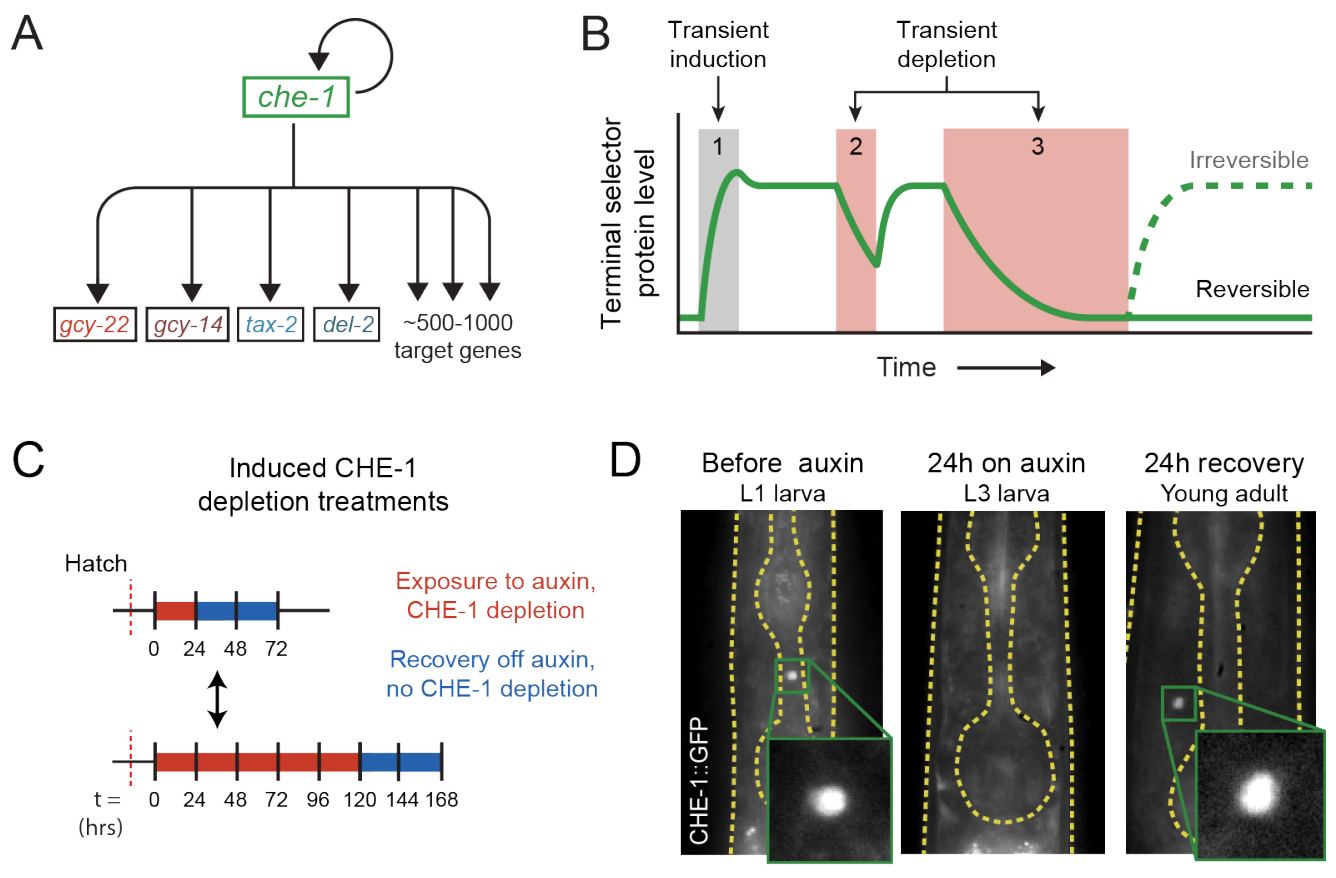

$E$

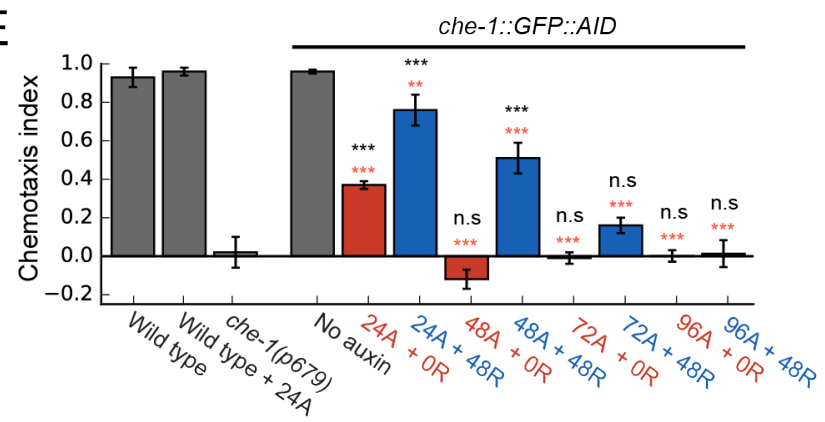

F 48h recovery after auxin

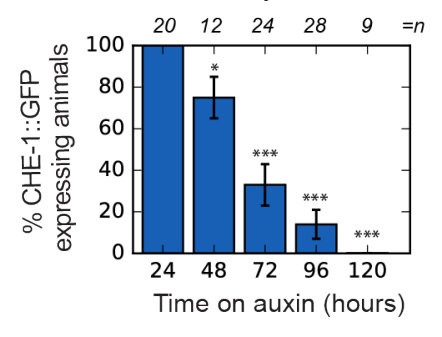

Figure 1. Loss of ASE neuron fate upon transient CHE-1 depletion.

(A) The terminal selector gene che-1 induces its own expression and that of 500-1000 target genes that together determine ASE neuron fate. Positive autoregulation of che-1 expression could result in bistable, switch-like behaviour. (B) Bistability generates sustained terminal selector expression upon transient induction (1), that is resilient to short periods of terminal selector depletion (2). However, bistable switches remain reversible and will lose terminal selector expression upon sufficiently long depletion (3), while irreversible switches will always recover. (C) Transient CHE-1 depletion using Auxin-Induced Degradation (AID). che$1:: G F P:: A I D$ L1 larvae (CHE-1::GFP) or young adults (chemotaxis) were exposed for different time periods to $1 \mathrm{mM}$ auxin to induce $\mathrm{CHE}-1$ degradation, and were subsequently characterized after a 24 or 48 hrs recovery period. (D) CHE-1::GFP fluorescence in che$1:: G F P:: A I D$ animals before (left) and after $24 \mathrm{hrs}$ auxin treatment (middle), and after a 
subsequent $24 \mathrm{hrs}$ recovery off auxin (right). Even though CHE-1::GFP is lost from ASE neurons after auxin treatment, it reappears after recovery off auxin. (E) Response to $10 \mathrm{mM}$ $\mathrm{NaCl}$ for wild-type animals, che-1(p679) mutants defective in $\mathrm{NaCl}$ chemotaxis, and che$1:: G F P:: A I D$ animals exposed to auxin for $24-96$ hrs $(24 \mathrm{~A}-96 \mathrm{~A})$ tested directly (OR) or after 48 hrs recovery (48R). che-1::GFP::AID animals on auxin showed a chemotaxis defect similar to che-1(p679) mutants. che-1::GFP::AID animals recovered chemotaxis to $\mathrm{NaCl}$ after 24 or $48 \mathrm{hrs}$ on auxin, but exhibited a persistent chemotaxis defect after sufficiently long, transient CHE-1::GFP::AID depletion. (F) Fraction of animals that recovered CHE$1:: G F P$ expression 48 hrs after auxin treatment of increasing length. No animals recovered CHE-1::GFP expression after 120 hrs of CHE-1 depletion. Error bars in E and F represent mean of 4 assays \pm S.E.M. ${ }^{*} \mathrm{P}<0.05$, ${ }^{* *} \mathrm{P}<0.01$, ${ }^{* *} \mathrm{P}<0.001$, in $\mathrm{E}$ significance is compared to che-1(p679) mutants (black) or no auxin (red). 
A

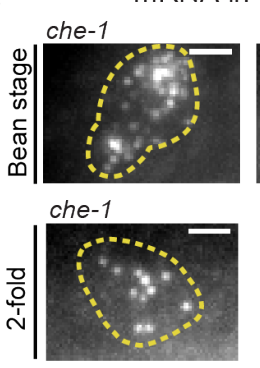

C
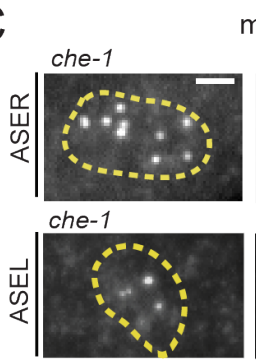

mRNA in L2 larvae

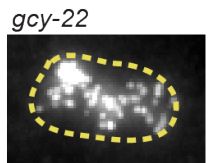
gcy-14

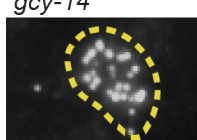

$E$

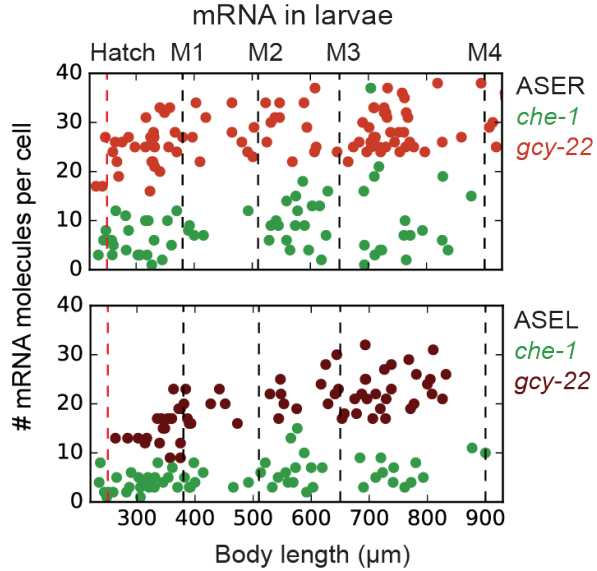

$\mathrm{B}$
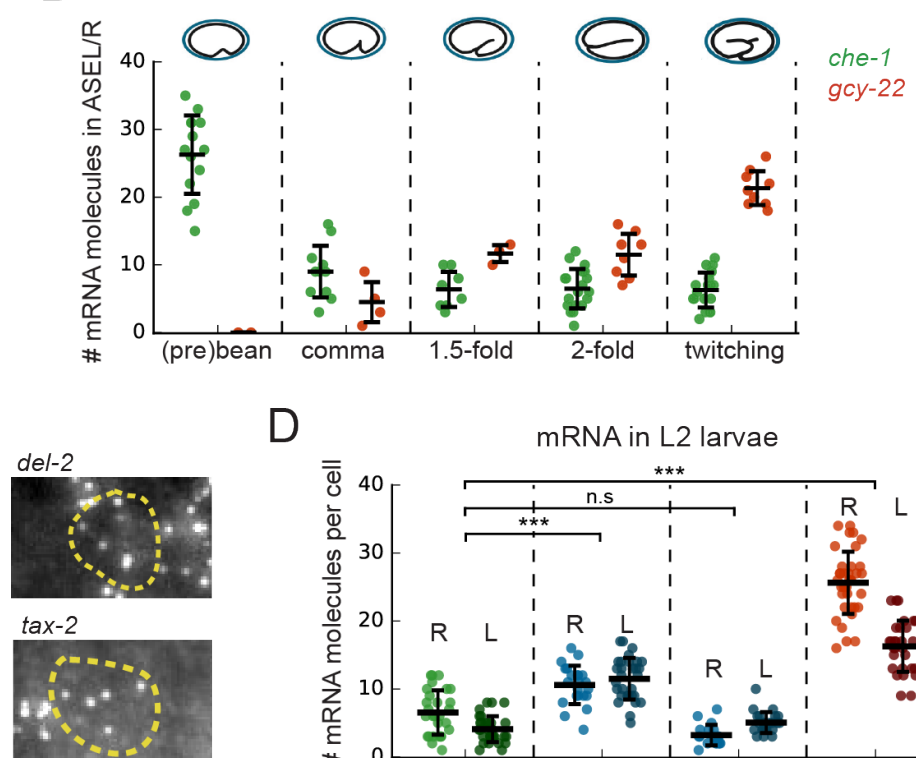

$\mathrm{D}$

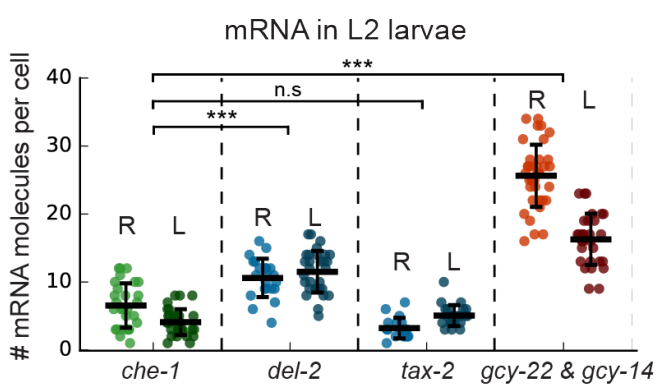

$\mathrm{F}$

CHE-1 protein in larvae
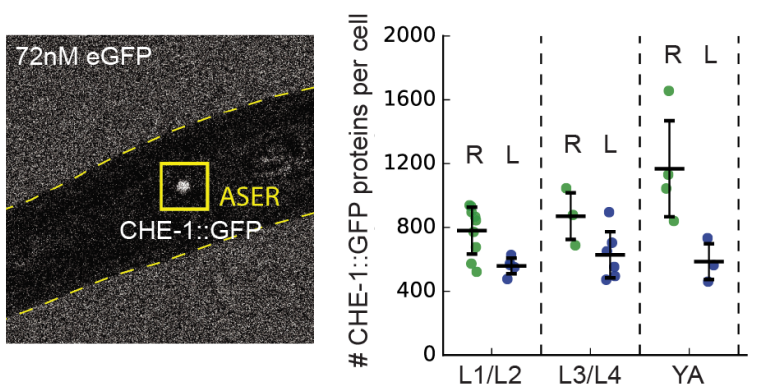

Figure 2. che-1 mRNA and protein copy numbers in ASE neurons.

(A) Expression of che-1 and the CHE-1 target gcy-22 in embryos at the bean (top) and 2fold stage (bottom). Each spot is a single mRNA molecule visualized by single molecule FISH (smFISH). Dashed lines outline ASE neuron cell bodies. Scale bar: $2 \mu \mathrm{m}$. (B) che-1 (green) and gcy-22 (red) mRNA levels during embryonic development. che-1 expression peaks during specification, but falls as expression of $\mathrm{CHE}-1$ target genes rise. (C) Expression of che-1 and CHE-1 targets gcy-22, gcy-14, tax-2 and del-2 visualized by smFISH in L2 larvae. Dashed lines outline left and right ASE neurons (ASEL/R). Scalebar: 2 um. (D) Quantification of expression of che-1 and CHE-1 targets in ASEL (L) and ASER (R) in L2 larvae. che-1 mRNA levels are low compared to other CHE-1 target genes. (E) Low 
che-1 expression (green, ASER/L) compared to expression of CHE-1 targets gcy-22 (red, ASER) and gcy-14 (red, ASEL) throughout larval development. Body length corresponds to developmental time, with approximate timing of hatching and molts between larval stages L1-L4 indicated by vertical lines. (F) Left panel: two-photon microscopy image of L2 larva expressing endogenously-tagged CHE-1::GFP, immersed in $72 \mathrm{nM}$ eGFP for calibration. Right panel: CHE-1::GFP protein molecules in ASER (green, R) and ASEL (blue, L) at different stages of post-embryonic development. The number of $\mathrm{CHE}-1$ proteins is comparable to the predicted number of $\mathrm{CHE}-1$ binding sites. Error bars in $\mathrm{B}, \mathrm{D}$ and $\mathrm{F}$ represent mean $\pm \mathrm{SD}$. ${ }^{* *} \mathrm{P}<0.001$. 

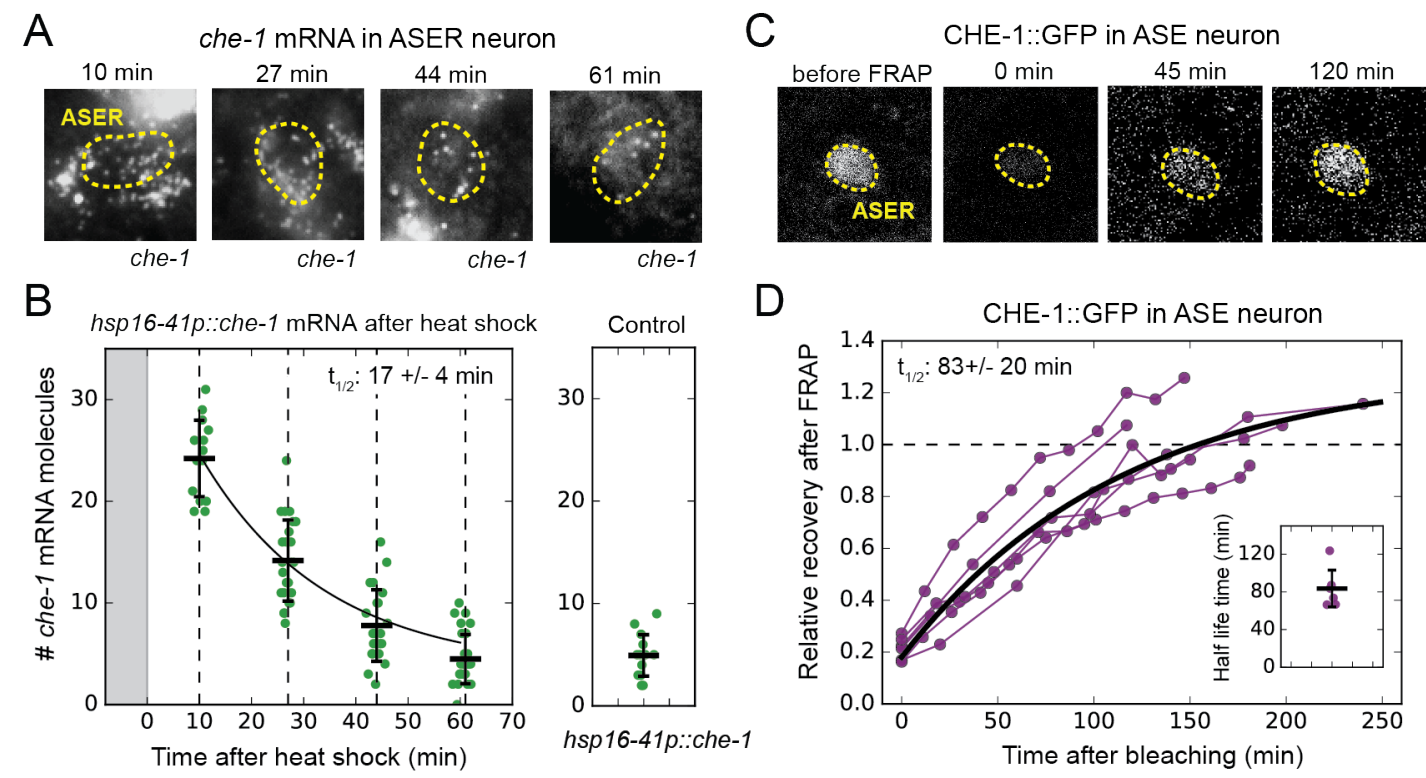

Figure 3. che-1 mRNA and protein lifetimes.

(A) che-1 mRNAs in L2 larvae at different times after induction of che-1 by a $37^{\circ} \mathrm{C}$ heat shock in hsp16-41p::che-1 animals, visualized by smFISH. Dashed lines outline ASER neuron. (B) che-1 mRNA level in ASE neurons of individual L2 animals (green) as function of time after a 10 min heat shock (grey area). Black line is the fitted decay curve. Control L2 larvae did not receive a heat shock. The measured che-1 mRNA half-life was $17 \pm 4$ mins. (C) CHE-1::GFP fluorescence recovery after photobleaching (FRAP) in the ASER neuron of a single L4 animal. Time is indicated relative to bleaching of CHE-1::GFP. (D) Fluorescence recovery of CHE-1::GFP in ASE neurons of $L 4$ or young adult animals $(n=6)$. An exponential recovery curve model was fitted to data of each individual animal (black line indicates the average recovery curve). The inset shows the fitted half-life for each individual animal. The average measured $\mathrm{CHE}-1:: \mathrm{GFP}$ protein half-life was $80 \pm 20 \mathrm{~min}$. Error bars in B represent mean $\pm S D$. $n \geq 10$ in $B$ and $n=6$ in $D$. 
A

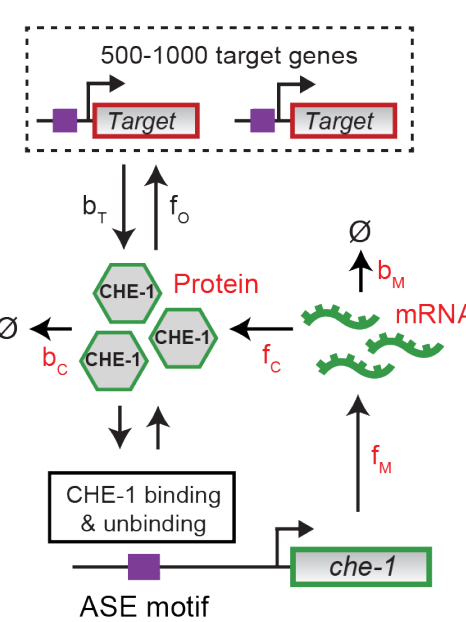

C

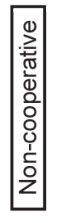

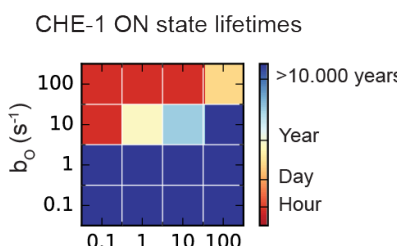

\begin{tabular}{|l|}
\hline \\
$\frac{\pi}{2}$ \\
$\frac{\pi}{0}$ \\
0 \\
0 \\
0
\end{tabular}

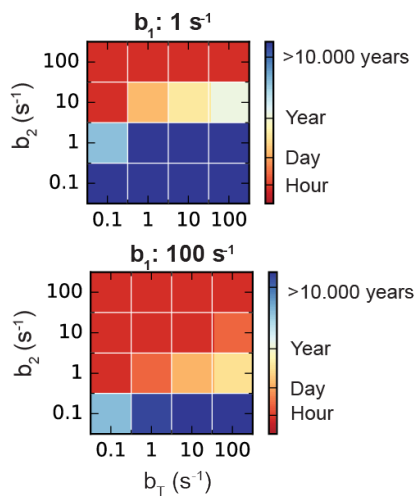

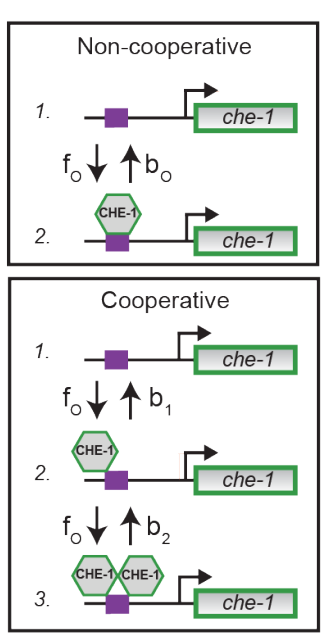

D High, medium and low stability
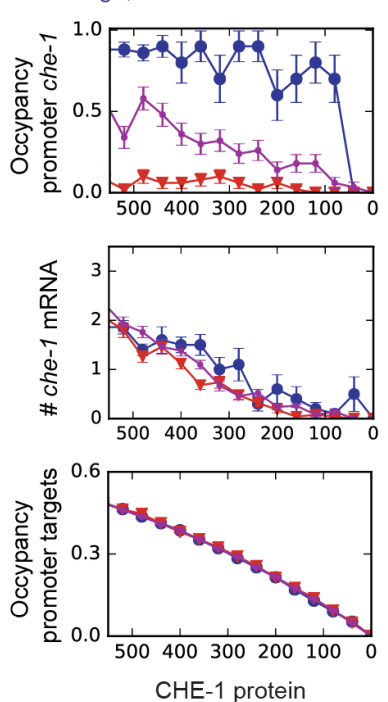

$\mathrm{CHE}-1$ protein
B
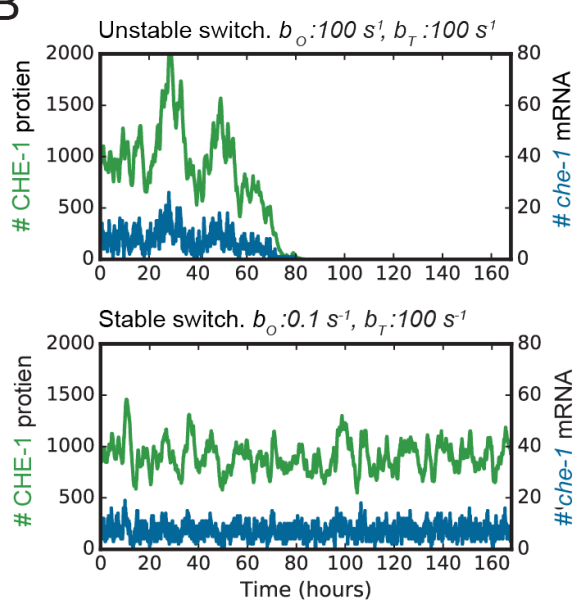

E
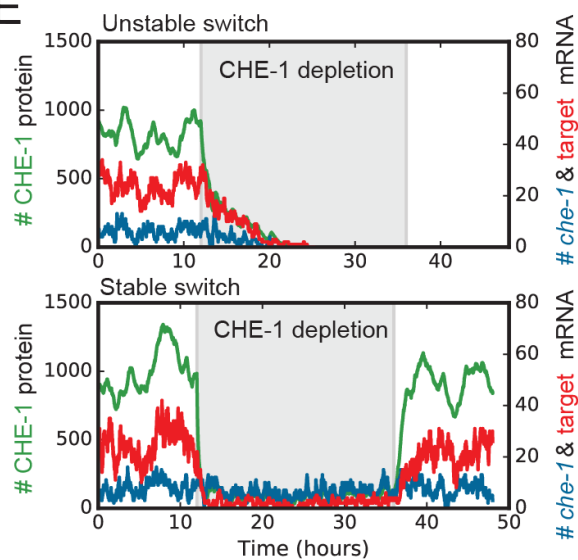

F

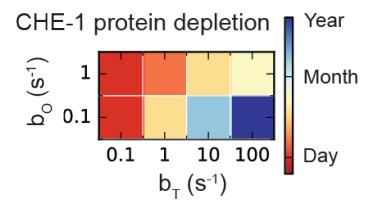

Figure 4. Stable ON state by preferential binding of CHE-1 at its own promoter.

(A) Overview of the bistable, stochastic CHE-1 switch model, including production and degradation of che-1 mRNA and protein, and binding of $\mathrm{CHE}-1$ protein to its own promoter and other target genes. Parameters constrained by experimental measurements are red. Inset: CHE-1 binding is modelled either as a monomer (non-cooperative) or as a dimer (cooperative). (B) Stochastic Gillespie simulations of the non-cooperative model for parameters with an unstable (top) or stable (bottom) ON state (che-1 expression), showing levels of che-1 mRNA (blue) and protein (green). For parameters resulting in an unstable switch, stochastic fluctuations induce a spontaneous transition to the OFF state (no che-1 expression). (C) Average ON state lifetimes calculated using Forward Flux Sampling (FFS) as function of the dissociation rates of $\mathrm{CHE}-1$ from its own promoter $\left(b_{O}\right.$ or $\left.b_{1}, b_{2}\right)$ and its 
target genes $\left(b_{T}\right)$ for the non-cooperative and cooperative model. Long $\mathrm{ON}$ state lifetimes occur for high che-1 promoter occupancy by CHE-1 $\left(b_{O}<1\right.$ or $\left.b_{1}, b_{2}<1\right)$ and preferential affinity of CHE-1 for its own promoter compared to that of its target genes $\left(b_{O} \ll b_{T}\right.$ or $\left.b_{2} \ll b_{T}\right)$. (D) Average CHE-1 occupancy of the promoter of che-1 (top) and other target genes (bottom), and average che-1 mRNA level (middle) during spontaneous transitions from the ON to the OFF state, as sampled by FFS. Shown are transition paths for parameters with low (red, $b_{O}=100 \mathrm{~s}^{-1}$ ), medium (magenta, $b_{O}=10 \mathrm{~s}^{-1}$ ), and high (blue, $b_{O}=1 s^{-1}$ ) stability of the ON state, with $b_{T}=10 s^{-1}$. For simulations with a stable ON state, the che-1 promoter remained fully occupied by $\mathrm{CHE}-1$, even as $\mathrm{CHE}-1$ protein levels approached zero, in contrast to the occupancy of promoters of other CHE-1 target genes. (E) Simulations showing the impact of transient depletion of $\mathrm{CHE}-1$ protein (green) on mRNA levels of che-1 (blue) and a target gene (red). CHE-1 is depleted to 100 molecules/cell by a transient increase in degradation ( $b_{C}$; grey region). For parameters with an unstable ON state (top), both che-1 and target gene mRNA levels fall rapidly, and do not recover when $\mathrm{CHE}-1$ depletion ceases. For a stable ON state (bottom), expression of che-1 is unaffected by $\mathrm{CHE}-1$ depletion, leading to full recovery once $\mathrm{CHE}-1$ depletion ends. (F) Average ON state lifetimes, calculated by FFS, during CHE-1 depletion to 100 molecules/cell. Parameter combinations with a stable ON state under normal conditions also maintain che-1 expression for hours or days under induced $\mathrm{CHE}-1$ depletion. 


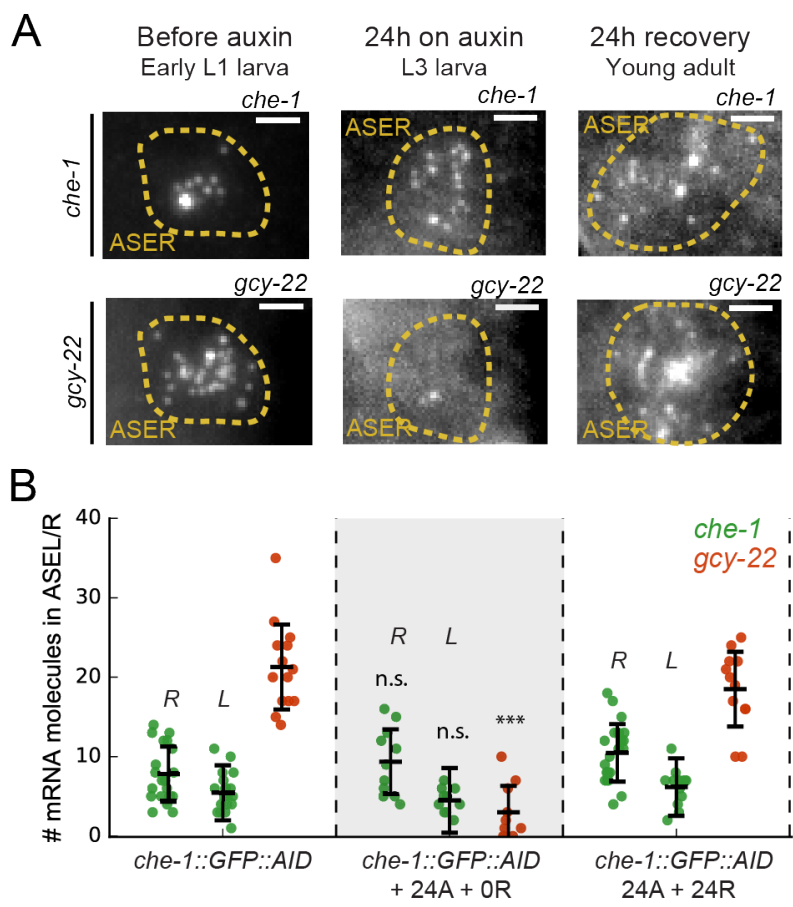

Figure 5. Maintenance of che-1 expression during transient CHE-1 depletion.

(A) che-1 and gcy-22 mRNA levels in ASER neurons, visualized by smFISH in che$1:: G F P:: A I D$ animals, at different moments in a $24 \mathrm{hrs}$ auxin and $24 \mathrm{hrs}$ recovery treatment. Scale bar: $2 \mu \mathrm{m}$. (B) che-1 (green, ASEL/R) and gcy-22 (red, ASER) mRNA levels quantified in che-1::GFP::AID animals at different times in a $24 \mathrm{hrs}$ auxin and $24 \mathrm{hrs}$ recovery treatment. Upon depletion of CHE-1 protein, che-1 expression was not impacted, while gcy22 levels strongly decreased. gcy-22 expression rose to wild-type levels after $24 \mathrm{hrs}$ recovery off auxin. Error bars in $B$ represent mean \pm SD. $n \geq 10$. ${ }^{* * *} P<0.001$. 
A
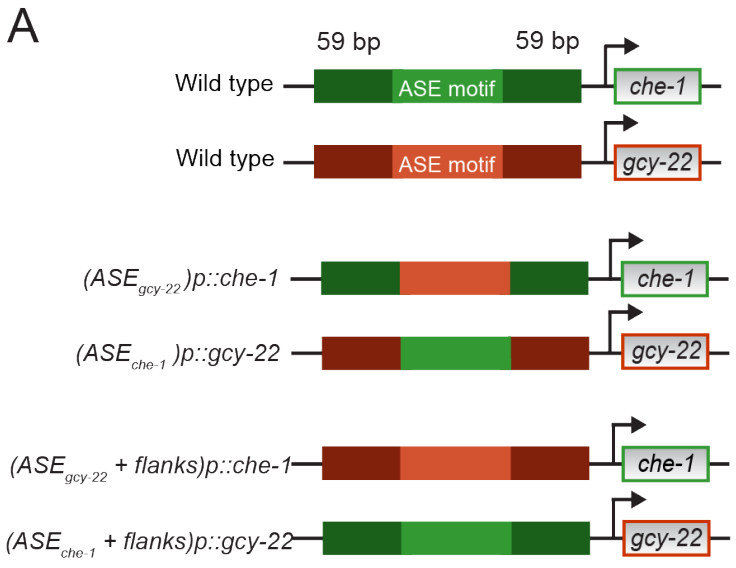

C

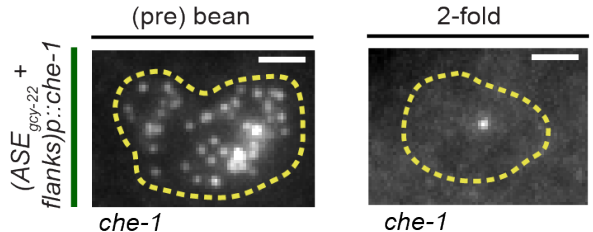

D

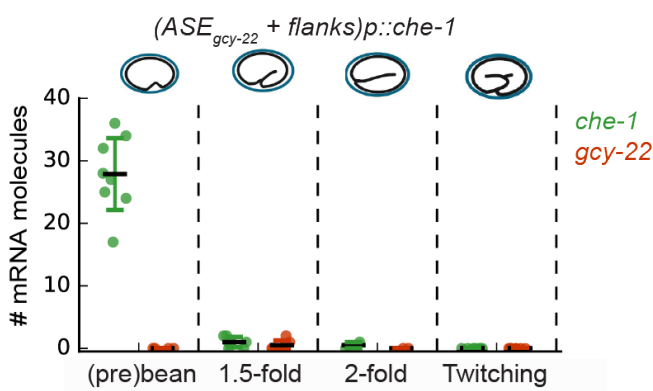

B

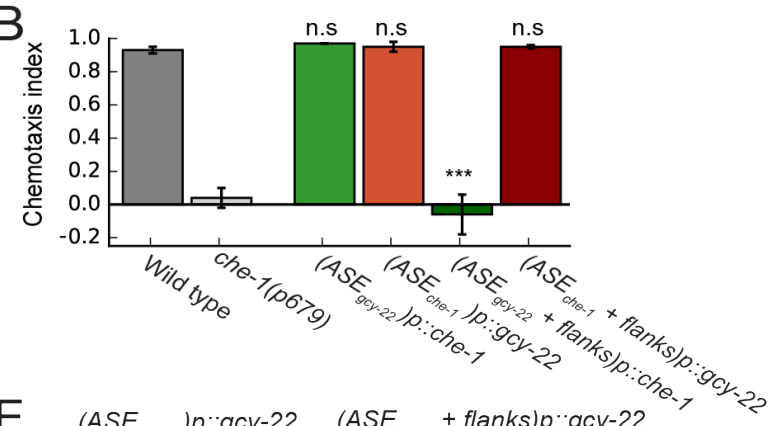

E

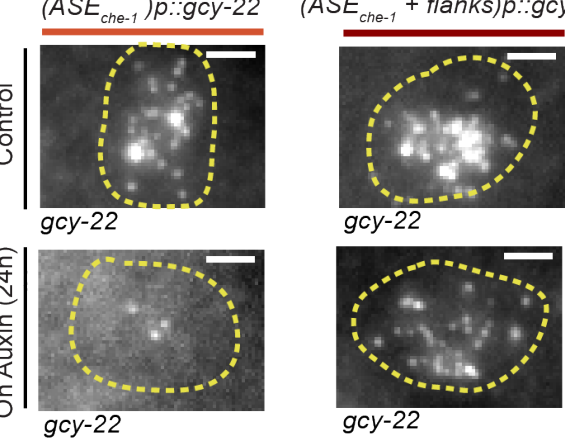

$\mathrm{F}$

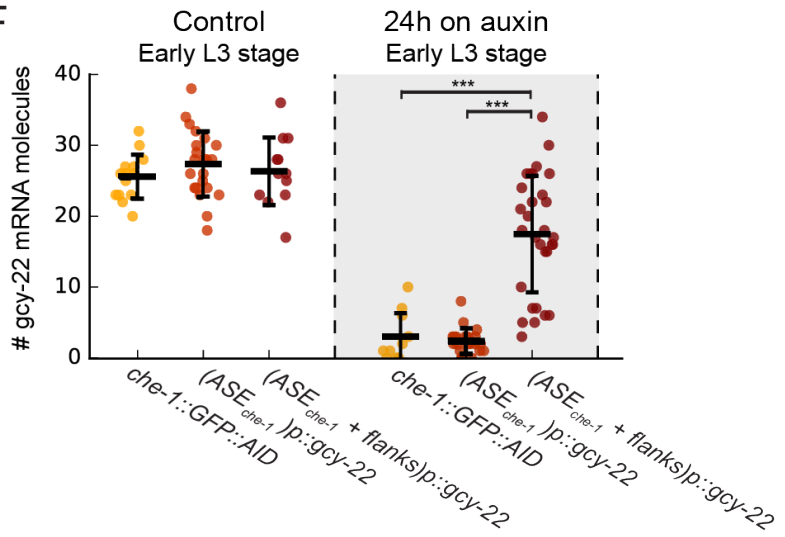

Figure 6. Region flanking CHE-1 binding site ensures resilience to CHE-1 depletion

(A) Overview of che-1 promoter mutants generated in the che-1::GFP:AID background. We exchanged either the che-1 (green) or gcy-22 (red) ASE motif, which binds CHE-1, or a larger region that includes $59 \mathrm{bp}$ flanks on either side. (B) Average chemotaxis index for response to $10 \mathrm{mM} \mathrm{NaCl}$, of wild-type and che-1(p679) animals, and che-1 promoter mutants. Exchange of ASE motifs between che-1 and gcy-22 promoters did not affect chemotaxis. Replacing the che-1 ASE motif with flanks for that of gcy-22 abolished chemotaxis to $\mathrm{NaCl}$; the reverse had no effect. (C) che-1 expression visualized by smFISH in ASE neurons of (ASE $\left.E_{g c y-22}+f l a n k s\right) p:: c h e-1$ embryos. In 2-fold embryos, che-1 mRNA levels were low. Scale bar: $1.5 \mu \mathrm{m}$. (D) che-1 and gcy-22 mRNA levels quantified in the ASE neurons of $\left(A S E_{g c y-22}+\right.$ flanks)p::che-1 embryos. After initial high che-1 expression at the time of ASE specification, che-1 and gcy-22 expression was almost absent, indicating a failure of ASE fate maintenance. (E) gcy-22 expression under normal conditions or upon CHE-1 
depletion by auxin, in L3 larvae of che-1 promoter mutants. Scale bar: $1.5 \mu \mathrm{m}$. (F) Quantification of gcy-22 mRNA levels upon auxin-induced CHE-1 depletion, in gcy-22 promoter mutants. In $\left(A S E_{g c y-22}\right) p:: c h e-1$ animals, gcy-22 mRNA levels fell on auxin, as observed before. However, in $\left.\left(A S E_{c h e-1}+f l a n k s\right) p:: g c y-22\right)$ animals treated with auxin $g c y-22$ levels remained high. Thus, the region flanking the che-1 ASE motif drives the maintenance of che-1 expression during $\mathrm{CHE}-1$ protein depletion. Error bars in $\mathrm{B}$ represent mean \pm S.E.M, D and F represent mean \pm SD. $n \geq 10$. ${ }^{* * *} P<0.001$. 
A

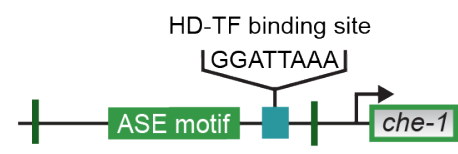

Otx-related HD-TF binding site

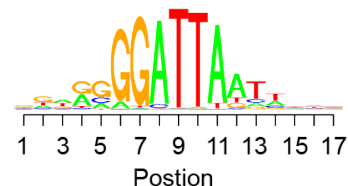

B

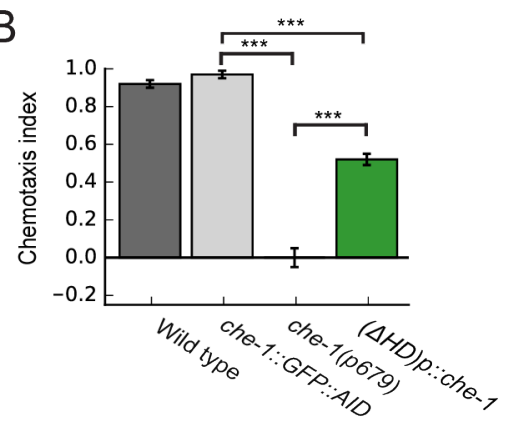

C

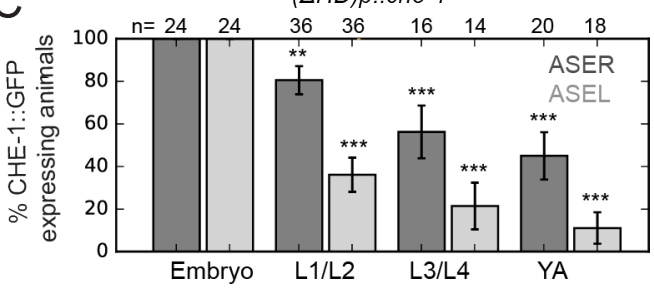

D
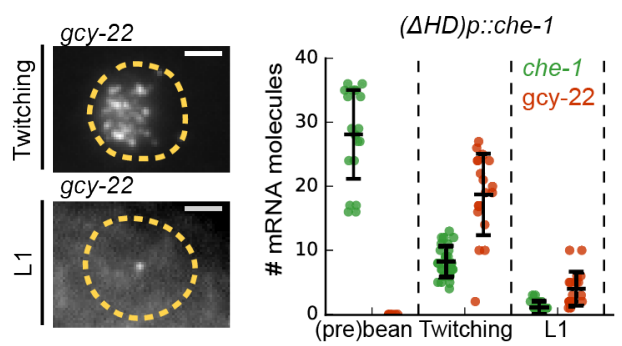

$E$
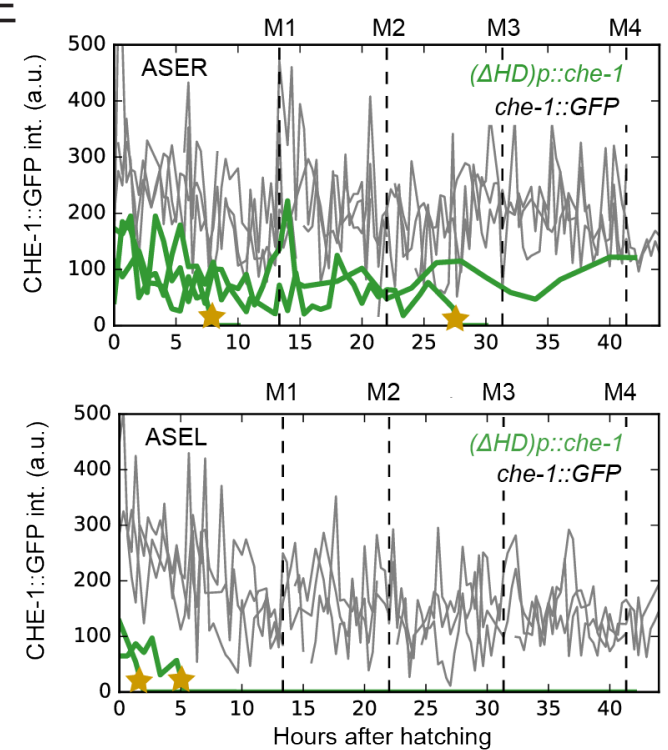

Figure 7. An Otx-related homeodomain transcription factor binding site involved in long-term maintenance of ASE cell fate.

(A) Position of an Otx-related homeodomain transcription factor (HD-TF) binding site in the che-1 promoter. Green lines indicate the positions of the $59 \mathrm{bp}$ flanks surrounding the ASE motif. HD-TF binding site depicted as a sequence logo. The HD-TF binding site is deleted in $(\Delta H D)$ p::che-1 animals. (B) Average chemotaxis index for response to $10 \mathrm{mM} \mathrm{NaCl}$, of wildtype, che-1::GFP::AID and che-1(p679) animals, and ( $\triangle H D) p:: c h e-1$ mutant. Deleting the $\mathrm{HD}-\mathrm{TF}$ binding site caused a decreased response to $\mathrm{NaCl}$ (C) Fraction of $(\Delta H D) p::$ che-1 animals expressing CHE-1::GFP in ASER (dark grey) and ASEL (light grey) at different developmental stages. CHE-1::GFP is progressively lost during development. (D) che-1 and gcy-22 mRNA levels in ( $\Delta H D) p:: c h e-1$ animals quantified by smFISH. Expression was similar to wild-type until late-stage, twitching embryos, but fell rapidly in newly-hatched L1 larvae. Scale bar: $2 \mu \mathrm{m}$. (E) CHE-1::GFP expression dynamics in single che-1::GFP (grey) and $(\Delta H D) p:: c h e-1$ (green) animals during larval development. Approximate timing of molts is indicated M1-M4. CHE-1::GFP expression in $(\Delta H D)$ p::che-1 animals was lost in a rapid and stochastic manner, at different times during development. Error bars represent S.E.M $(B, D)$ or S.D. (C). $n \geq 10 .{ }^{* *} P<0.01,{ }^{* * *} P<0.001$. 


\section{STAR methods}

\section{Experimental model and subject details}

C. elegans strains and handling

The following alleles were used in this study: che-1(ot856[che-1::GFP]) (kindly provided by Dylan Rahe from the Hobert lab) (Leyva-Diaz and Hobert 2019), gcy-5(ot835[gcy5::SL2::mNeonGreen]) II; otTi6[hsp16-41p::che-1::2xFLAG] X, che-1(p679), ieSi57[Peft3::TIR1::mRuby::unc-54 3'UTR, Cbr-unc-119(+)] II. A complete list of strains used in this study, including transgenic strains generated by CRISPR/Cas9, are listed in the Key Resources Table.

The wild-type strain used was the C. elegans variety Bristol, strain N2. All C. elegans strains were maintained on Nematode Growth Medium (NGM) plates, containing E. coli strain OP50 as a food source, at $20^{\circ} \mathrm{C}$ (Brenner 1974), unless indicated otherwise. Worms were maintained according to standard protocol.

\section{Method details}

CRISPR/Cas9-mediated genome editing

Genome editing was performed according to protocol (Dokshin et al. 2018) and using ssODN repair templates with 35 bp homology arms (Paix et al. 2017). An AID tag was endogenously inserted at the C-terminus of GFP in a che-1(ot856[che-1::GFP]) background using guide g2 and a repair template containing the degron sequence (Zhang et al. 2015), generating che-1(gj2089[che-1::GFP::AID]). Subsequently, the ieSi57 allele (Zhang et al. 2015) was introduced. All che-1 or gcy-22 promoter mutations were made in this che1(gj2089[che-1::GFP::AID];ieSi57 background. (ASE $E_{g c y-22}+$ flanks)p::che-1 was generated using a template containing the ASE motif of the $g c y-22$ promoter and its 59 bp flanking regions, and guides g51 and g52. (ASE $E_{\text {che-1 }}+$ flanks)p::gcy-22 was generated using a template containing the ASE motif of che-1 and its 59 bp flanks and guides g55 and g56. $\left(A S E_{g c y-22}\right) p:: c h e-1$ was generated using a template containing the ASE motif of gcy-22 and guide g53. ( $\left.A S E_{\text {che-1 }}\right) p:: g c y-22$ was generated using a template containing the ASE motif of che-1 and guide g57. ( $\triangle H D) p:: c h e-1$ was generated using a template containing the $35 \mathrm{bp}$ flanking regions of the HD motif from the che-1 promoter and guide g54. Guides and ssODNs used in this research are listed in Table S4.

\section{Single Molecule Fluorescence In Situ Hybridization (smFISH)}

The oligonucleotides for the smFISH probe sets were designed with optimal GC content and specificity for the gene of interest using the Stellaris RNA FISH probe designer. The 
oligonucleotides were synthesized with a $3^{\prime}$ amino C7 modification and purified by LGC Biosearch Technologies. Conjugation of the oligonucleotides with either Cy5 (GE Amersham) or Alexa594 (Invitrogen) was done as previously described (a). Sequences of each probe set are listed in Table S3 (with exception of $g c y-14$ ). The smFISH protocol was performed as previously described (Raj et al. 2008; Ji and van Oudenaarden 2012). Briefly, staged animals were washed from plates with $\mathrm{M} 9$ buffer and fixed in $4 \%$ formaldehyde in $1 \mathrm{x}$ PBS, gently rocking at room temperature (RT) for 40 minutes (young adults for 35 minutes). Fixation of embryos required a snap-freeze step to crack the eggshells by submerging embryos, after 15 minutes in fixation buffer, in liquid nitrogen, and thawing on ice for 20 minutes. After fixation, the animals were $2 x$ washed with 1 xPBS and resuspended in $70 \%$ ethanol overnight at $4^{\circ} \mathrm{C}$. Ethanol was removed and animals were washed with $10 \%$ formamide and $2 \mathrm{X}$ SSC, as preparation for the hybridization. Animals were incubated with the smFISH probes overnight in the dark at $37^{\circ} \mathrm{C}$ in a hybridization solution (Stellaris) with added $10 \%$ formamide. The next day, animals were washed $2 x$ with $10 \%$ formamide and $2 X$ SSC each with an incubation step of 30 minutes at $37^{\circ} \mathrm{C}$. The last wash step contains DAPI $5 \mu \mathrm{g} / \mathrm{mL}$ for nuclear staining. The wash buffer was removed, and animals were resuspended in 2X SSC and stored at $4^{\circ} \mathrm{C}$ until imaging. The $2 \mathrm{X} \mathrm{SSC}$ was aspirated and animals were immersed in $100 \mu \mathrm{l}$ GLOX buffer (0.4\% glucose, $10 \mathrm{mM}$ Tris- $\mathrm{HCl}, \mathrm{pH}$ 8.0, 2X SSC) together with $1 \mu \mathrm{l}$ Catalase (Sigma-Aldrich) and $1 \mu$ l glucose oxidase (Sigma-Aldrich) $(3.7 \mathrm{mg} / \mathrm{mL}$ ) to prevent bleaching during imaging.

Microscopy images of smFISH samples were acquired with a Nikon Ti-E inverted fluorescence microscope, equipped with a 100X plan-apochromat oil-immersion objective and an Andor Ikon-M CCD camera controlled by $\mu$ Manager software (Edelstein et al. 2014). smFISH analysis was performed with custom Python software, based on a previously described method (Raj et al. 2008). Briefly, we first convolved the smFISH images with a Gaussian filter. Next, candidate spots were selected via manual thresholding, and partially overlapping spots were separated via finding $3 \mathrm{D}$ regional intensity maxima. We used the spatial localization of $g c y-22$ or gcy-14 mRNA molecules which are highly expressed ASEspecific genes, to estimate the cell boundaries of the ASE neurons. The coverage of the ASE cell bodies with gcy-22 or gcy-14 mRNA molecules agreed with GFP markers (gcy$5 p:: G F P$ or $g c y-7 p:: G F P)$ that marked the cell body.

\section{CHE-1 protein quantification}

Staged che-1::GFP knock-in animals were bathed in $72 \mathrm{nM}$ and $48 \mathrm{nM}$ eGFP recombinant protein (Bio-connect) with $0.25 \mathrm{mM}$ Levamisole (Sigma-Aldrich) in M9 buffer in a glass chambered cover glass systems (IBL baustoff), which were coated with $0.5 \mathrm{mg} / \mathrm{ml}$ kappa- 
capsein in the buffer MRB80 (80 mM Pipes, $4 \mathrm{mM} \mathrm{MgCl2,} 1 \mathrm{mM} \mathrm{EGTA,} \mathrm{pH} 6.8$ with $\mathrm{KOH}$ ) to prevent binding of eGFP to the cover glass and chamber walls. Images of bathed che1::GFP animals in eGFP solution were acquired with a Nikon Eclipse Ti inverted microscope, equipped with a Nikon C1 confocal scan head, a $100 \mathrm{~mW}$ Argon ion laser (488nm, Coherent), and a S Fluor 40×1.3 NA and an Apo TIRF 100×1.49 NA objective. Calibration of eGFP with che-1::GFP animals was repeated 2 times at different days with $72 \mathrm{nM}$ and 48 nM eGFP concentrations, of which we took the average calibration measurements. For ease of measuring, the CHE-1::GFP signal of animals was measured with the exact same microscope and software settings, except placing the animals submersed in $0.25 \mathrm{mM}$ Levamisole (Sigma-Aldrich) in M9 buffer on agar pads with the same cover glass thickness on top. The ASE neuron closest to the cover glass was imaged in larvae to circumvent tissue scattering. Embryos were followed in time (at $22^{\circ} \mathrm{C}$ ) and imaged every 20 minutes with the exact same microscope and software settings, from bean stage until twitching started. For both larvae and embryos, the slices focused at the approximate middle of the ASE neuron nuclei were used for quantifying the CHE-1::GFP signal. The volumes of the nuclei were calculated by measuring the radii of the nuclei in $x, y$ and $z$ direction from the CHE-1::GFP signal with the assumption that the nucleus shape resembles a ellipsoid, using the following equation: $V=\frac{4}{3} \pi x y z$.

\section{FRAP}

To estimate the protein degradation rate of CHE-1::GFP, we used Fluorescence Recovery After Photobleaching (FRAP). Animals were immobilized using Polybead microspheres (Polyscience) in M9 buffer on agarose pads covered with a cover glass. To prevent dehydration of animals, the coverslip was sealed with VALAP (vaseline, lanolin and paraffin, ratio $1: 1: 1)$. Animals were monitored at several time points during the experiment if they were still alive by checking very subtle movement and/or pumping behaviour. The GFP signal in the ASE neurons of the animals was bleached until approximately $20 \%$ of the initial signal was left. After bleaching, the GFP signal was measured every 20 or 30 minutes until the signal had recovered. Images were taken with the same microscope as in the CHE-1 protein quantification section. We measured for each time point the average GFP intensity in the ASE neurons and subtracted the background intensity measured nearby the ASE neurons. The degradation rate was calculated from the initial slope of the growth curve using the following exponential model: $R(t)=\left(\frac{f}{b}\right)+\left(x_{0}-\left(\frac{f}{b}\right)\right) e^{-b t}$, where $x_{0}$ is the initial fluorescent intensity at the start of the recovery curve right after bleaching. $b$ and $f$ represent the $\mathrm{CHE}-1$ protein degradation and production rate, which are fitted on the individual measured recovery curves, to obtain the average $\mathrm{CHE}-1$ protein degradation rate. 
che-1 mRNA stability

We induced che-1 mRNA overexpression with the otTi6 [hsp16-41p::che-1::2xFLAG] $X$ inducible heat shock strain, and we quantified with smFISH che-1 mRNA in the ASER neurons in L2 larvae over time $\left(t_{i}=0, \ldots, 4\right) \sim 17$ min apart until recovery. We determined the relative amount of che-1 mRNAs from the start of the measurement, $n\left(t_{i}\right)=\frac{N\left(t_{i}\right)-N_{\text {control }}}{N_{H S}-N_{\text {control }}}$, where $N_{\text {control }}$ is the calculated average amount of che-1 mRNAs when there is no che-1 mRNA overexpression, $N_{H S}$ the calculated average amount of che-1 mRNAs at the first measured time point right after heat shock induction, and $n(t)$ is the amount of che-1 mRNAs at the three remaining time points. An exponential degradation curve $e^{-a t}$ was fitted to the experimentally determined values $n\left(t_{i}\right)$, to obtain the approximate che-1 mRNA degradation rate.

\section{Mathematical Model of the CHE-1 switch}

\section{Overview cooperative model}

The cooperative mathematical model assumes that $\mathrm{CHE}-1$ protein $(C)$ has to bind as a dimer on the che-1 promoter to induce che-1 mRNA expression. The binding and unbinding of the two CHE-1 proteins at the che-1 promoter $(O)$ is separated into two events, first one CHE-1 binds with binding rate $f_{O}$ and unbinds with unbinding rate $b_{1}$ from the che-1 promoter $(O C)$. When the first CHE-1 protein is bound to the che-1 promoter, the second $\mathrm{CHE}-1$ protein binds with the same binding rate $f_{O}$ next to the first $\mathrm{CHE}-1$, forming a dimer on the che-1 promoter (OC2), and unbinds with the unbinding rate $b_{2}$. CHE-1 proteins bind as a monomer on the target gene promoters $\left(O_{T} C\right)$, with the unbinding rate $b_{T}$, and with same the binding rate $f_{O}$ as on the che-1 promoter. che-1 mRNA $(M)$ is transcribed with the production rate $f_{M}$ only when two $\mathrm{CHE}-1$ proteins are bound to the che-1 promoter, and che-1 mRNA is translated into CHE-1 protein with the protein production rate $f_{C}$. Both che-1 mRNA and CHE-1 protein are degraded with the degradation rates, $b_{M}$ and $b_{C}$ respectivily. This leads to the following differential equations:

$$
\begin{gathered}
\frac{d O C}{d t}=f_{O}\left(O^{*}-(O C+O C 2)\right) C-b_{1} O C \\
\frac{d O C 2}{d t}=f_{O} O C \cdot C-b_{2} O C 2 \\
\frac{d O_{T} C}{d t}=f_{O}\left(O_{T}^{*}-O_{T} C\right) C-b_{T} O_{T} \cdot C
\end{gathered}
$$




$$
\begin{gathered}
\frac{d M}{d t}=f_{M} O C 2-b_{M} M \\
\frac{d C}{d t}=-f_{O}\left(O^{*}-(O C+O C 2)\right) C-f_{O} O C \cdot C-f_{O}\left(O_{T}^{*}-O_{T} C\right) C+b_{1} O C+b_{2} O C 2+b_{T} O_{T} \cdot C+f_{C} M-b_{C} C
\end{gathered}
$$

Where $O^{*}$ is the total number of che-1 promoters and where $O_{T}^{*}$ is the total number of target genes.

\section{Overview non-cooperative model}

In the non-cooperative model, CHE-1 binds $\left(f_{O}\right)$ and unbinds $\left(b_{O}\right)$ as a monomer on the che-1 promoter $(O C)$ and induces che-1 expression as a monomer. The other reactions in the model are the same as in the cooperative model. This leads to the following differential equations:

$$
\begin{gathered}
\frac{d O C}{d t}=f_{O}\left(O^{*}-O C\right) C-b_{O} O C \\
\frac{d O_{T} C}{d t}=f_{O}\left(O_{T}^{*}-O_{T} C\right) C-b_{T} O_{T} \cdot C \\
\frac{d M}{d t}=f_{M} O C-b_{M} M \\
\frac{d C}{d t}=-f_{O}\left(O^{*}-O C\right) C-f_{O}\left(O_{T}^{*}-O_{T} C\right) C+b_{O} O C+b_{T} O_{T} C+f_{C} M-b_{C} C
\end{gathered}
$$

\section{Bistability}

In the cooperative model $\mathrm{CHE}-1$ proteins bind as a dimer at the che-1 promoter to induce che-1 expression. The binding of two CHE-1 proteins in the system, implies non-linear behaviour, giving rise to bistability. We have in the cooperative model three fixed points, 2 stable and 1 unstable fixed point. The 2 stable fixed points represent the so-called "ON state" (high CHE-1) and the "OFF state" (low CHE-1) of the CHE-1 switch. When the switch is in the OFF state, it has to cross the unstable point to reach the ON state. In contrast, the non-cooperative model, in which $\mathrm{CHE}-1$ proteins bind as a monomer at the che-1 promoter to induce che-1 expression, has only two fixed points. The first fixed point represents the $\mathrm{ON}$ state, in which $\mathrm{CHE}-1$ protein levels are high. The second fixed point is a half-stable point, when there is no CHE-1 protein, the switch is OFF. However, the introduction of, for 
example, only 1 che-1 mRNA in the system would be sufficient to turn the switch from the OFF state to the ON state.

\section{Simulations}

To study the short-term lifetime ( $<1$ weeks) of the cooperative and non-cooperative CHE-1 switch, we performed stochastic Gillespie simulations on both models (Gillespie 1977). We used a custom written python script to simulate the reactions involved in both CHE-1 switch models. All reactions describing the differential equations from the cooperative and noncooperative model, are summarized in Fig. 3A. Parameters remain unchanged during simulations (with exception for transient depletion simulations) and species are initiated in the ON state.

To study ON state lifetimes of the CHE-1 switch exceeding 1 weeks, in the cooperative and non-cooperative CHE-1 switch model, we used Forward Flux Sampling (Allen et al. 2006; Allen et al. 2009), a computational method which allowed us to estimate lifetimes of CHE-1 switches. It was not necessary to integrate pruning into the algorithm since this would not result in improvement in computational efficiency. The FFS algorithm was initiated with the same initial conditions as the Gillespie simulations. Interfaces of the FFS algorithm were chosen according to the variance of $\mathrm{CHE}-1$ protein in the $\mathrm{ON}$ state, to generate a $5-10 \%$ chance of $\mathrm{CHE}-1$ protein trajectories crossing the first interface (with an exception for very unstable switch, where $\mathrm{CHE}-1$ protein simulations immediately run to the OFF state). The typical step size of the interfaces was 20 and the number of interfaces was between 20 and 35.

\section{Parameters}

We can divide the parameters in the following groups: 1.) experimentally determined parameters, 2.) parameters that we could approximate, 3.) unconstrained parameters and 4.) parameters that we could calculate with help of the other parameters. First, the parameters which were experimentally determined. We based the che-1 mRNA degradation rate $\left(b_{M}\right)$ on direct measurement of che-1 mRNA degradation that we quantified in the ASER neuron (Fig 2A-B). We used an approximation of che-1 mRNA lifetime of 20 minutes. The amount of CHE-1 protein $(C)$ was set to 900 molecules (average of ASER and ASEL at L4/YA stage) based on the CHE-1 protein quantification experiments, and the amount of che-1 mRNAs $(M)$ was set to 7 molecules (average of ASER and ASEL at L4 stage) based on the smFISH experiments in wild-type animals. 
The binding rate of $\mathrm{CHE}-1$ at its own promoter $f_{O}$ and the target gene promoters $f_{O}$ is approximated by the diffusion limited binding rate. The diffusion limited binding rate of CHE-1 on promoter sites was calculated using the following diffusion equation: $f_{D}=4 \pi \sigma D$, where reaction cross section $\sigma=1 \cdot 10^{-2} \mu \mathrm{m}$, i.e. the size of the promoter binding site, and the diffusion coefficient constant is $D=1 \mu \mathrm{m}^{2} \mathrm{~s}^{-1}$. To obtain the diffusion coefficient that we can apply in our simulations, which includes information about the volume of the nucleus, the diffusion coefficient $\left(f_{D}\right)$ was divided with the average nucleus volume $\left(V_{C}\right)$ of $4 \mu \mathrm{m}^{3}$ in ASER and ASEL, resulting in a diffusion limited binding rate of $f_{O}=\frac{f_{D}}{V_{C}}=0.03 \mathrm{~s}^{-1}$.

To approximate the number of target sites where the $\mathrm{CHE}-1$ protein can bind, we used the crude approximation depicted from the study of Etchberger et al. (2007), in which they found 596 genes represented with at least three times as many tags in the ASE versus AFD SAGE library, which is expected to contain almost no "false positives". We acquired the total amount of ASE motifs (minimum score of $98 \%$ ) in each of the promoter regions $(<1000$ bp upstream of the start site) with a custom written $R$ script, using the TFBSTools/JASPAR2018 packages, resulting in a total of 425 ASE motifs. However, the data set lacked, for example, housekeeping genes expressed in the ASE neurons that could be under control of che-1. The sci-RNA-seq dataset from the study of Cao et al. provides information on genes expected to be expressed in the ASE neurons, including ASE nonspecific genes (Cao et al. 2017). A total of 1400-1500 genes are expected to be expressed in the ASEL and ASER (score $>100$, removal of "false-positives"), resulting in $~ 1000$ ASE motifs on which CHE-1 could potentially bind. In the simulations we used either 500 or 1000 target sites.

The unbinding rates of $\mathrm{CHE}-1$ from its own promoter and the target gene promoters, $b_{1}, b_{2}, b_{T}$, are the only unconstrained parameters, and are ranged between 0.1 and $100 \mathrm{sec}^{-1}$ in the FFS simulations. The $\mathrm{CHE}-1$ protein degradation rate $b_{C}$, the $\mathrm{CHE}-1$ production rate $f_{C}$ and the che-1 mRNA production rate $f_{M}$ are dependent on the unbound fraction of CHE1 protein. To calculate the three unknown rates $b_{C}, f_{C}, f_{M}$, first the $\mathrm{CHE}-1$ protein degradation rate $b_{C}$ was set at the experimentally measured parameter, and used to calculate the che-1 mRNA production rate $f_{M}$ and $\mathrm{CHE}-1$ protein production rate $f_{C}$. The $\mathrm{CHE}-1$ production rate is influenced by the amount of $\mathrm{CHE}-1$ bound at the target gene promoters, hence why the $\mathrm{CHE}-1$ protein production and degradation had to be fitted to the experimentally measured CHE-1 protein FRAP curve. The fitting was done by introducing CHE-1::GFP species, bleached or unbleached, in the existing model in order to reproduce in simulation the FRAP experiments. The bleached CHE-1::GFP proteins could also bind and unbind the promoters 
and induce che-1 expression, but the bleached CHE-1::GFP proteins could not be produced, only degraded.

In the table below, we summarize all parameter values. In this table, all parameter values are defined for the Unstable CHE-1 switch (4B), and the other model parameter values are only given when they deviate from those used in the Unstable CHE-1 switch (4B).

\begin{tabular}{|c|c|c|}
\hline Parameter & Description & Value \\
\hline $\begin{array}{l}\text { Unstable switch } \\
\text { (4B) }\end{array}$ & Unstable CHE-1 switch, average lifetime of $\sim 10$ days & \\
\hline$C$ & Number of $\mathrm{CHE}-1$ protein molecules & 900 \\
\hline$M$ & Number of che-1 mRNA molecules & 7 \\
\hline$O_{S}$ & Number of che-1 promoters & 1 \\
\hline$O_{T_{-} S}$ & Number of target genes & 500 \\
\hline$f_{O}$ & Binding rate of $\mathrm{CHE}-1$ on che-1 or target gene promoters & $0.03 \mathrm{sec}^{-1}$ \\
\hline$b_{O}$ & Unbinding rate of $\mathrm{CHE}-1$ from che-1 promoter & $100 \mathrm{sec}^{-1}$ \\
\hline$b_{T}$ & Unbinding rate of $\mathrm{CHE}-1$ from target gene promoters & $100 \sec ^{-1}$ \\
\hline$f_{M}$ & che-1 mRNA production rate & $0.0302 \mathrm{sec}^{-1}$ \\
\hline$b_{M}$ & che-1 mRNA degradation rate & $0.00083 \mathrm{sec}^{-1}$ \\
\hline$f_{C}$ & $\mathrm{CHE}-1$ protein production rate & $0.0274 \mathrm{sec}^{-1}$ \\
\hline$b_{C}$ & $\mathrm{CHE}-1$ protein degradation rate & $0.00024 \mathrm{sec}^{-1}$ \\
\hline Stable switch (4B) & $\begin{array}{l}\text { Highly stable switch, slower unbinding rate of CHE-1 from } \\
\text { its own promoter }\end{array}$ & \\
\hline$b_{O}$ & Unbinding rate of $\mathrm{CHE}-1$ from che-1 promoter & $0.1 \mathrm{sec}^{-1}$ \\
\hline$f_{M}$ & che-1 mRNA production rate & $0.0059 \mathrm{sec}^{-1}$ \\
\hline$f_{C}$ & $\mathrm{CHE}-1$ protein production rate & $0.0261 \mathrm{sec}^{-1}$ \\
\hline$b_{C}$ & $\mathrm{CHE}-1$ protein degradation rate & $0.00023 \mathrm{sec}^{-1}$ \\
\hline Stable switch (4E) & Highly stable switch, depleted to $100 \mathrm{CHE}-1$ & \\
\hline$b_{O}$ & Unbinding rate of $\mathrm{CHE}-1$ from che-1 promoter & $0.1 \mathrm{sec}^{-1}$ \\
\hline$f_{M}$ & che-1 mRNA production rate & $0.0059 \mathrm{sec}^{-1}$ \\
\hline$b_{C}$ & $\mathrm{CHE}-1$ protein degradation rate & $0.0019 \mathrm{sec}^{-1}$ \\
\hline$f_{C}$ & $\mathrm{CHE}-1$ protein production rate & $0.024 \mathrm{sec}^{-1}$ \\
\hline$b_{P}$ & Target mRNA degradation rate & $0.0008 \mathrm{sec}^{-1}$ \\
\hline$f_{P}$ & Target mRNA production rate & $0.09 \mathrm{sec}^{-1}$ \\
\hline $\begin{array}{l}\text { Unstable switch } \\
\text { (4E) }\end{array}$ & Unstable switch, depleted to $100 \mathrm{CHE}-1$ & \\
\hline$b_{O}$ & Unbinding rate of $\mathrm{CHE}-1$ from che-1 promoter & $10 \sec ^{-1}$ \\
\hline$b_{T}$ & Unbinding rate of $\mathrm{CHE}-1$ from target gene promoters & $10 \sec ^{-1}$ \\
\hline$b_{C}$ & $\mathrm{CHE}-1$ protein degradation rate & $0.00076 \mathrm{sec}^{-1}$ \\
\hline$f_{C}$ & $\mathrm{CHE}-1$ protein production rate & $0.026 \mathrm{sec}^{-1}$ \\
\hline$b_{P}$ & Target mRNA degradation rate & $0.0004 \mathrm{sec}^{-1}$ \\
\hline$f_{P}$ & Target mRNA production rate & $0.016 \mathrm{sec}^{-1}$ \\
\hline
\end{tabular}

The parameters for the depletion simulations panel (Fig 4E) include an extra promoter $(P O)$ with production and degradation rates of $(P M)$ mRNA, $b_{P}, f_{P}$, to simulate the production of target mRNA induced by $\mathrm{CHE}-1$. The binding and unbinding rates of the extra promoter are the same as for the target genes, $f_{O}, b_{T}$. 
If spontaneous switches from the ON to the OFF state occur as a Poisson process with rate $r$, then the probability of a switching event occurring at time $t$ is given by the exponential distribution $p(t)=r \exp (-r t)$. The fraction of animals in which a switching event occurs before time $t=T$ is given by the cumulative distribution $P(t)=1-\exp (-r t)$. This fraction is smaller than a value $\phi$ if $r<-\ln (1-\phi) / T$. For small fractions $\phi \approx 0$, this can be approximated as $r<\phi / T$. If a switching event can occur only in 1 out of $10^{6}$ animals, $\phi=1 \cdot 10^{-6}$, within the average animal lifetime of $T=2$ weeks, then the required life time of the ON state is given by $1 / r>2.3 \cdot 10^{5}$ years.

\section{Auxin induced protein degradation}

The Auxin Inducible Degron (AID) System was employed as previously described (Zhang et al. 2015). Animals were initially grown on NGM plates with OP50 without IAA (SigmaAldrich). To induce degradation of CHE-1, L1 staged animals were transferred to NGM plates with OP50 and $1 \mathrm{mM} I A A$ in $\mathrm{EtOH}$ at $20^{\circ} \mathrm{C}$.

For the CHE-1::GFP::AID measurements, the duration of auxin exposure and recovery were varied in each treatment. The animals were transferred every other day to new NGM plates with $1 \mathrm{mM}$ IAA or recovery NGM plates without IAA to prevent mixing of generations. Each treatment contained a control group of che-1::GFP::AID animals never exposed to auxin. For imaging, the animals were placed on an $5 \%$ agarose pad submerged in $0.25 \mathrm{mM}$ Levamisole (Sigma-Aldrich) in M9 with a cover glass on top. We checked for CHE-1::GFP::AID in the ASE neurons with a wide field microscope. Older animals showed stronger autofluorescence in the head, giving rise to nuclei-like structures which could be confused with ASE neurons. The GFP (FITC) channel was combined with the TRITC channel to correct for the autofluorescence (Teuscher and Ewald 2018). Due to tissue scattering and decrease in CHE-1::GFP signal in old animals, we found the ASE neuron closest to the cover glass was most reliable. The control group always showed CHE$1:: G F P:: A I D$ in the ASE neurons: even though CHE-1::GFP::AID levels decreased with age, even 10 days old animals had well identifiable ASE neurons.

\section{$\mathrm{NaCl}$ chemotaxis assay}

The quadrant assay used to asses chemotaxis to $\mathrm{NaCl}$ was adapted from Wicks et al. and Jansen et al. (Wicks et al. 2000; Jansen et al. 2002). Briefly, two diagonally opposite quadrants of a sectional petri dish (Star Dish, Phoenix Biomedical) were filled with $13.5 \mathrm{~mL}$ buffered agar (1.7\% Agar, $5 \mathrm{mM} \mathrm{K} \mathrm{HPO}_{4} / \mathrm{KH}_{2} \mathrm{PO}_{4} \mathrm{pH}$, $1 \mathrm{mM} \mathrm{CaCl}$ and $1 \mathrm{mM} \mathrm{MgSO}$ ) containing $\mathrm{NaCl}$ and two diagonally opposite quadrants with $13.5 \mathrm{~mL}$ buffered agar without 
$\mathrm{NaCl}$. Immediately before the assay, the plastic dividers between the quadrants were covered with a thin layer of agar. Age synchronized young adult $C$. elegans populations were washed 3 times for 5 min with CTX buffer $\left(5 \mathrm{mM} \mathrm{K}_{2} \mathrm{HPO}_{4} / \mathrm{KH}_{2} \mathrm{PO}_{4} \mathrm{pH}\right.$, $1 \mathrm{mM} \mathrm{CaCl}$ and $1 \mathrm{mM} \mathrm{MgSO}_{4}$ ). Approximately 100 animals were placed in the middle of a sectional dish. After $10 \mathrm{~min}$, animals on each quadrant were counted and a chemotaxis index $(\mathrm{Cl})$ was calculated for each plate $(\mathrm{Cl}=(\#$ animals on $\mathrm{NaCl}-\#$ animals not no $\mathrm{NaCl}) /$ total \# animals $)$. To determine the $\mathrm{Cl}$ of a strain, 2 assays per day were performed on at least 2 different days.

To assess the effect of $\mathrm{CHE}-1$ depletion on chemotaxis, animals were bleached and cultured for $24 \mathrm{hrs}$ on NGM plates without IAA. After $24 \mathrm{hrs}$ animals were transferred to NGM plates containing $1 \mathrm{mM}$ IAA. To remove eggs and larvae, animals were thoroughly rinsed using CTX buffer and a $30 \mu \mathrm{m}$ pluriStrainer (pluriSelect) and transferred to a fresh NGM plate, with our without $1 \mathrm{mM}$ IAA, starting at $96 \mathrm{hrs}$ into the experiment and repeated every $24 \mathrm{hrs}$ until the end of the experiment. After the experimental treatment duration, the chemotaxis index was determined. Subsequently, recovery was started by transferring animals to NGM plates without IAA and the chemotaxis index was determined $24 \mathrm{hrs}$ and 48 hrs thereafter. At each timepoint, the chemotaxis index was determined in similarly aged che-1(p679) and untreated che-1::GFP::AID animals.

\section{Time lapse}

Time-lapse imaging was performed as previously described (Gritti et al. 2016). Briefly, micro chambers are made out of polyacrylamide hydrogel, made from a $10 \%$ dilution of 29:1 acrylamide/bis-acrylamide (Sigma-Aldrich) was mixed with $0.1 \%$ ammonium persulfate (Sigma-Aldrich) and $0.01 \%$ TEMED (Sigma-Aldrich) as polymerization initiators. For the time-lapse experiments the chambers were $240 \times 240 \times 20 \mu \mathrm{m}$, these dimensions were sufficient to contain enough OP50 bacteria to sustain development until animals started laying eggs.

We used a Nikon Ti-E inverted microscope with a 40X objective in all experiments. The microscope has a Hamamatsu Orca Flash 4.0 v2 camera set at full frame and full speed. The camera chip is $13 \times 13 \mathrm{~mm}$ and contains $4 \mathrm{Mp}$. We used $488 \mathrm{~nm}$ lasers (Coherent OBIS-LS 488-100) for fluorescence excitation. We used a high fluorescent signal of 100 $\mathrm{mW}$ with an exposure time of $10 \mathrm{~ms}$, since the fluorescent signal of CHE-1::GFP is relatively low. To keep track of the molting cycle as indication of the age of the animals, we used 
bright field imaging, which contained a red LED (CoolLED, 630nm). Time-lapse images were acquired every 20 minutes without detectable phototoxicity effects.

Images were analysed with custom written time-lapse software, and with ImageJ. Briefly, first we corrected the raw images for experimental aberrations with flat and dark field images acquired at the end of the experiment. For quantification purposes, we computed the average fluorescence of each ASE neuron via drawing a region of interest around each nucleus and we corrected the average intensity by subtracting the background level close to the ASE neuron.

\section{Quantification and statistical analysis}

Image analysis of CHE-1::GFP quantification and intensity measurements was performed with the ImageJ distribution Fiji (Schindelin et al. 2012). For the quantification data shown in graphs of all figures, the dots represent individual values, the boxplots without box represent the mean and the standard deviation.

Statistical analyses were performed either using R software, version 3.6.0, or with Python 3.5 Package SciPy. Comparisons of the chemotaxis indexes were performed using a oneway ANOVA, followed by a pairwise t-test with Holm correction. Significance between control versus conditions in smFISH data were preformed using one-way ANOVA, followed by a Tukey multiple comparison test. Significance of \% expression of CHE-1::GFP in ASE neurons between treatment groups (on auxin) and control group (no auxin) was preformed using Fisher exact test.

\section{Data and Code Availability Statement}

Data and software in this paper are available upon request to the Lead Contact.

\section{Key resources table}




\section{SI figure legends}

A

Chemotaxis quadrant assay with $10 \mathrm{mM} \mathrm{NaCl}$

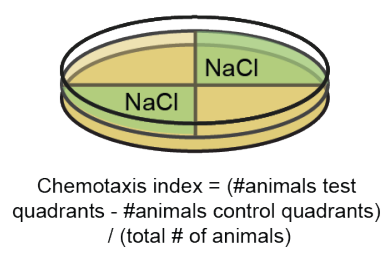

C

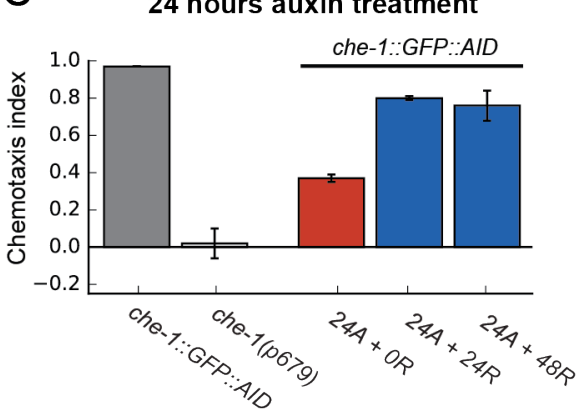

72 hours auxin treatment

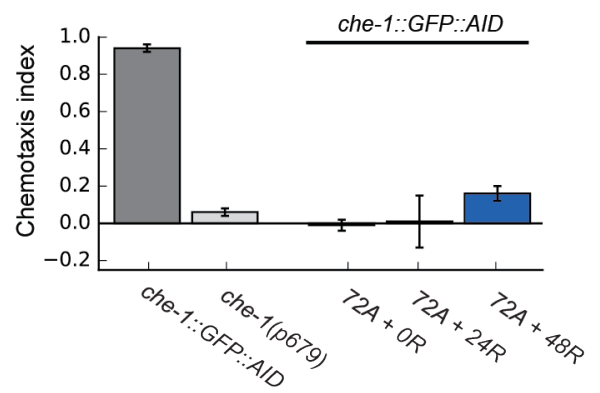

B

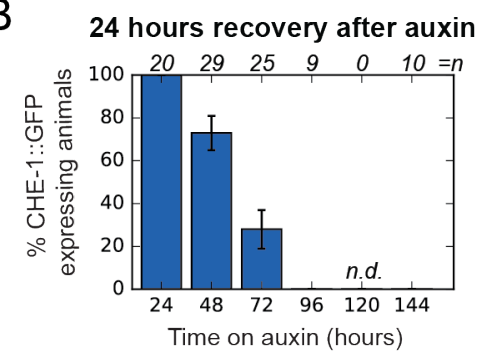

48 hours auxin treatment

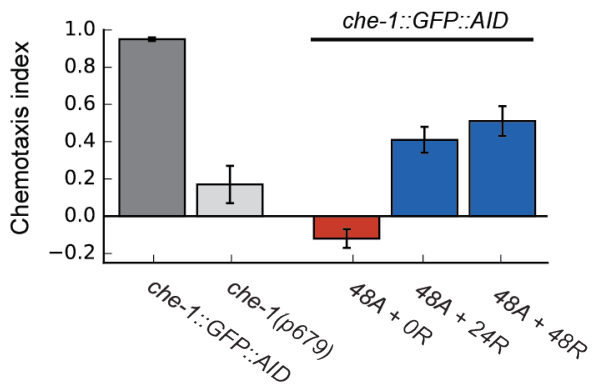

96 hours auxin treatment

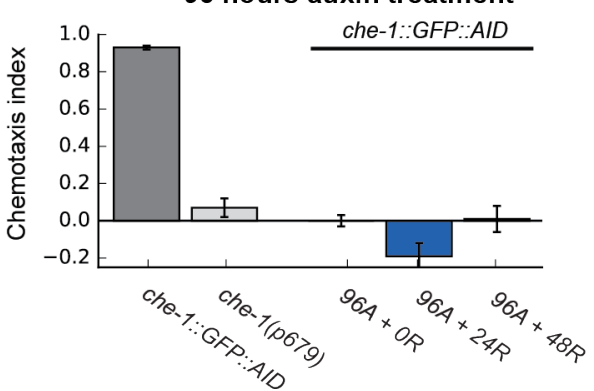

Figure S1. CHE-1 depletion leads to loss of ASE cell fate. (A) Schematic of quadrant chemotaxis assay and chemotaxis index $(\mathrm{Cl})$ calculation. (B) Percentage of animals that recovered CHE-1::GFP expression after auxin treatment of increasing length with a recovery period of 24 hours. (C) Chemotaxis index for wild-type, che-1(p679) and che-1::GFP::AID animals exposed to auxin for 24-96 hrs after a recovery period of 0,24 or $48 \mathrm{hrs}$. 

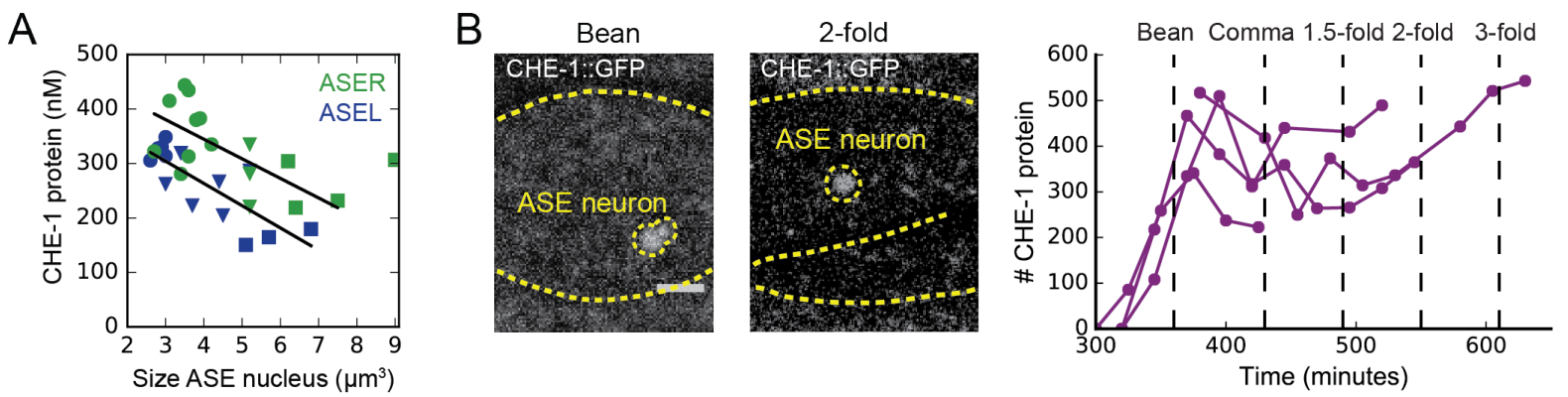

Figure S2. CHE-1 copy numbers in larvae and embryos. (A) CHE-1 protein concentration in ASER (green) and ASEL (blue) at different stages of post-embryonic development (L1/L2: circles, L3/L4: triangle, young adult: squares). CHE-1 protein concentration decreases with the age of the animals. (B) Images of che-1::GFP embryos for quantification of CHE-1::GFP in ASE neurons with confocal microscopy (left). Number of CHE-1 protein molecules over time in embryos, starting at the bean stage until twitching started (at $22^{\circ} \mathrm{C}$ ) (right). Embryos showed similar levels of CHE-1 protein as the L 1 larvae. 
A Cooperative switch, target genes: 500

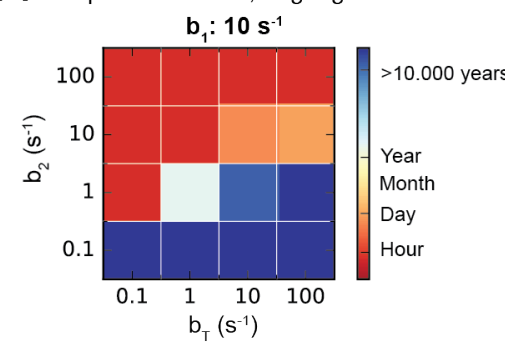

$\mathrm{B}$

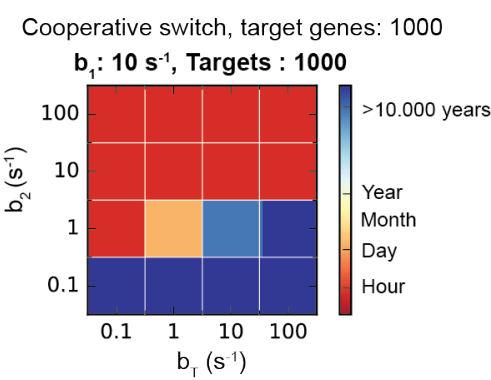

C
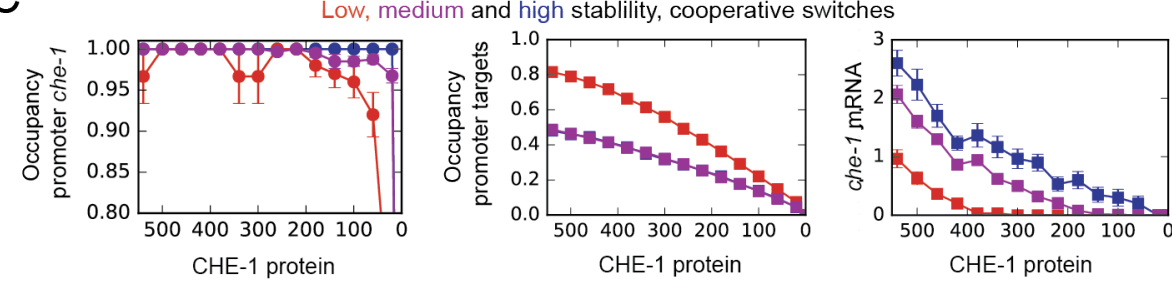

D Cooperative switch, CHE-1 depletion

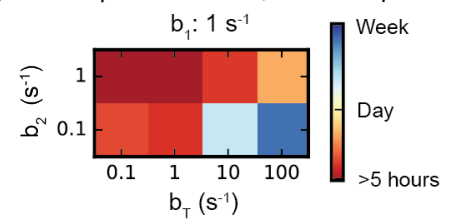

Figure S3. Dependence of ON state stability on number of targets and cooperativity. $(A, B)$ Average ON state lifetimes calculated using Forward Flux Sampling (FFS) as function of the dissociation rates of CHE-1 from its own promoter $\left(b_{2}, b_{1}\right)$ and target genes $\left(b_{T}\right)$ promoters, for the cooperative model with $O_{T}^{*}=500$ (A) or 1000 (B) CHE-1 targets. ON state lifetimes are lower for 1000 targets, but long lifetimes of many years are still found. $b_{1}=10$ $\sec ^{-1}$ and $b_{1}, b_{T}$ varied between $0.1-100 \mathrm{sec}^{-1}$. (C) Average CHE-1 occupancy of the promoter of che-1 (left) and other target genes (middle), and average che-1 mRNA level (right) during spontaneous transitions from the ON to the OFF state, as sampled by FFS, for the cooperative model. Shown are transition paths for parameters with low (red, $b_{2}=100 \mathrm{~s}^{-1}, b_{T}=10 \mathrm{~s}^{-1}$ ), medium (magenta, $b_{2}=10 \mathrm{~s}^{-1}, b_{1}=100 \mathrm{~s}^{-1}$ ), and high (blue, $b_{2}=1 s^{-1}, b_{1}=100 s^{-1}$ ) stability of the ON state, with $b_{1}=10 s^{-1}$. (D) Average ON state lifetimes calculated using Forward Flux Sampling (FFS) of the cooperative model during depletion of $\mathrm{CHE}-1$ protein, as function of the dissociation rates of $\mathrm{CHE}-1$ from its own promoter $\left(b_{2}\right)$ and its target genes $\left(b_{T}\right)$, where $b_{1}=10 \mathrm{sec}^{-1}$. 

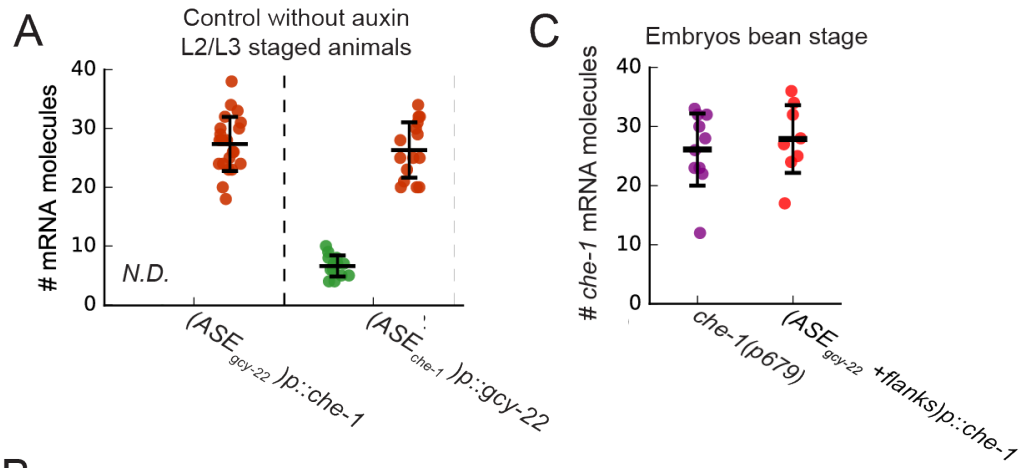

B

$\left(A S E_{g c y-22}+\right.$ flanks)p::che-1

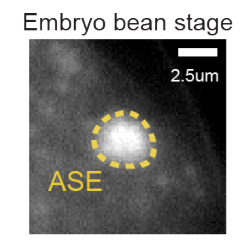

CHE-1::GFP in bean stage CHE-1::GFP in larvae: $0 / 12$

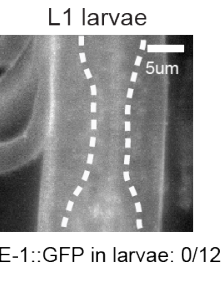

Figure S4. Controls for promoter region mutants. (A) Quantification of gcy-22 (red) and che-1(green) mRNA levels in $\left(A S E_{\text {gcy-22 }}\right) p:: c h e-1$ and $\left(A S E_{\text {che-1 }}\right) p:: g c y-22$ L2/L3 animals without auxin revealed wild type mRNA levels for both genes. (B) CHE-1::GFP expression in $\left(A S E_{g c y-22}+\right.$ flanks)p::che-1 bean stage embryos and L1 larvae. Embryos showed CHE$1::$ GFP expression (5/5 animals), whereas L1 larvae no longer expressed CHE-1::GFP (0/12 animals). (C) (ASE $E_{g c y-22}+$ flanks)p::che-1 embryos showed similar levels of che-1 mRNA during the bean stage as the che-1(p679) mutant. 
A

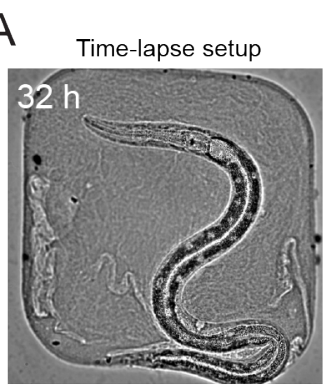

CHE-1::GFP in ASER in $(\triangle H D) p:: c h e-1$ animal
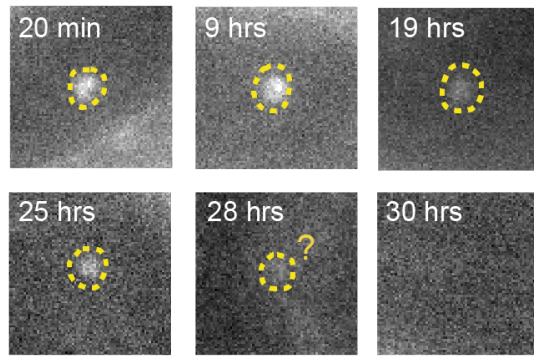

$30 \mathrm{hrs}$

B

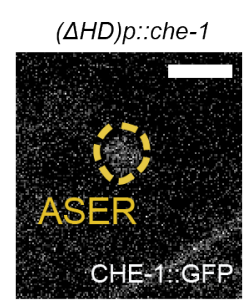

L1 larvea

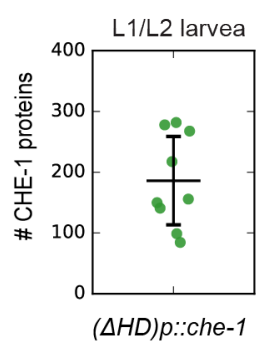

Figure S5. Time lapse imaging and quantification of CHE-1::GFP in ( $\triangle H D) p:: c h e-1$ animals. (A) Long-term time-lapse microscopy of a single $(\Delta H D) p:: c h e-1$ animal, using microchambers to constrain larvae to the field of view (left). CHE-1::GFP expression in ASER disappeared rapidly in the L3 larval stage, $30 \mathrm{hrs}$ after hatching (right). (B) Image of CHE-1::GFP in $(\Delta H D) p::$ che-1 L1 larva (left). Quantification of CHE-1::GFP protein in $(\Delta H D) p:: c h e-1 \mathrm{~L} 1 / \mathrm{L} 2$ staged animals (right). 


\section{References}

Acar, M., A. Becskei, and A. van Oudenaarden. 2005. 'Enhancement of cellular memory by reducing stochastic transitions', Nature, 435: 228-32.

Allen, R. J., D. Frenkel, and P. R. ten Wolde. 2006. 'Forward flux sampling-type schemes for simulating rare events: efficiency analysis', J Chem Phys, 124: 194111.

Allen, R. J., C. Valeriani, and P. Rein Ten Wolde. 2009. 'Forward flux sampling for rare event simulations', J Phys Condens Matter, 21: 463102.

Alon, U. 2007. 'Network motifs: theory and experimental approaches', Nat Rev Genet, 8: 450-61.

Anderson, P. 1995. 'Mutagenesis', Methods Cell Biol, 48: 31-58.

Brenner, S. 1974. 'The genetics of Caenorhabditis elegans', Genetics, 77: 71-94.

Cao, J., J. S. Packer, V. Ramani, D. A. Cusanovich, C. Huynh, R. Daza, X. Qiu, C. Lee, S. N. Furlan, F. J. Steemers, A. Adey, R. H. Waterston, C. Trapnell, and J. Shendure. 2017. 'Comprehensive single-cell transcriptional profiling of a multicellular organism', Science, 357: 661-67.

Chang, S., R. J. Johnston, Jr., and O. Hobert. 2003. 'A transcriptional regulatory cascade that controls left/right asymmetry in chemosensory neurons of C. elegans', Genes Dev, 17: 2123-37.

Coburn, C. M., and C. I. Bargmann. 1996. 'A putative cyclic nucleotide-gated channel is required for sensory development and function in C. elegans', Neuron, 17: 695-706.

Deneris, E. S., and O. Hobert. 2014. 'Maintenance of postmitotic neuronal cell identity', Nat Neurosci, 17: 899-907.

Dhondt, I., V. A. Petyuk, S. Bauer, H. M. Brewer, R. D. Smith, G. Depuydt, and B. P. Braeckman. 2017. 'Changes of Protein Turnover in Aging Caenorhabditis elegans', Mol Cell Proteomics, 16: 1621-33.

Dokshin, G. A., K. S. Ghanta, K. M. Piscopo, and C. C. Mello. 2018. 'Robust Genome Editing with Short Single-Stranded and Long, Partially Single-Stranded DNA Donors in Caenorhabditis elegans', Genetics, 210: 781-87.

Edelstein, A. D., M. A. Tsuchida, N. Amodaj, H. Pinkard, R. D. Vale, and N. Stuurman. 2014. 'Advanced methods of microscope control using muManager software', J Biol Methods, 1.

Etchberger, J. F., E. B. Flowers, R. J. Poole, E. Bashllari, and O. Hobert. 2009. 'Cisregulatory mechanisms of left/right asymmetric neuron-subtype specification in $\mathrm{C}$. elegans', Development, 136: 147-60.

Etchberger, J. F., A. Lorch, M. C. Sleumer, R. Zapf, S. J. Jones, M. A. Marra, R. A. Holt, D. G. Moerman, and O. Hobert. 2007. 'The molecular signature and cis-regulatory architecture of a C. elegans gustatory neuron', Genes Dev, 21: 1653-74.

Ferrell, J. E., Jr. 2002. 'Self-perpetuating states in signal transduction: positive feedback, double-negative feedback and bistability', Curr Opin Cell Biol, 14: 140-8.

Fornasiero, E. F., S. Mandad, H. Wildhagen, M. Alevra, B. Rammner, S. Keihani, F. Opazo, I. Urban, T. Ischebeck, M. S. Sakib, M. K. Fard, K. Kirli, T. P. Centeno, R. O. Vidal, R. U. Rahman, E. Benito, A. Fischer, S. Dennerlein, P. Rehling, I. Feussner, S. Bonn, M. Simons, H. Urlaub, and S. O. Rizzoli. 2018. 'Precisely measured protein lifetimes in the mouse brain reveal differences across tissues and subcellular fractions', Nat Commun, 9: 4230.

Gebhardt, J. C., D. M. Suter, R. Roy, Z. W. Zhao, A. R. Chapman, S. Basu, T. Maniatis, and X. S. Xie. 2013. 'Single-molecule imaging of transcription factor binding to DNA in live mammalian cells', Nat Methods, 10: 421-6.

Gillespie, Daniel T \%J The journal of physical chemistry. 1977. 'Exact stochastic simulation of coupled chemical reactions', 81: 2340-61.

Gregor, T., D. W. Tank, E. F. Wieschaus, and W. Bialek. 2007. 'Probing the limits to positional information', Cell, 130: 153-64. 
Gritti, N., S. Kienle, O. Filina, and J. S. van Zon. 2016. 'Long-term time-lapse microscopy of C. elegans post-embryonic development', Nat Commun, 7: 12500.

Hench, J., J. Henriksson, A. M. Abou-Zied, M. Luppert, J. Dethlefsen, K. Mukherjee, Y. G. Tong, L. Tang, U. Gangishetti, D. L. Baillie, and T. R. Burglin. 2015. 'The Homeobox Genes of Caenorhabditis elegans and Insights into Their Spatio-Temporal Expression Dynamics during Embryogenesis', PLoS One, 10: e0126947.

Hobert, O. 2008. 'Regulatory logic of neuronal diversity: terminal selector genes and selector motifs', Proc Natl Acad Sci U S A, 105: 20067-71.

Hobert, O. 2010. 'Neurogenesis in the nematode Caenorhabditis elegans', WormBook: 1-24.

Hobert, O. 2016. 'Terminal Selectors of Neuronal Identity', Curr Top Dev Biol, 116: 455-75.

Hobert, O., and P. Kratsios. 2019. 'Neuronal identity control by terminal selectors in worms, flies, and chordates', Curr Opin Neurobiol, 56: 97-105.

Hsiao, H. Y., D. Jukam, R. Johnston, and C. Desplan. 2013. 'The neuronal transcription factor erect wing regulates specification and maintenance of Drosophila R8 photoreceptor subtypes', Dev Biol, 381: 482-90.

Jansen, G., D. Weinkove, and R. H. Plasterk. 2002. 'The G-protein gamma subunit gpc-1 of the nematode C.elegans is involved in taste adaptation', EMBO J, 21: 986-94.

Ji, N., and A. van Oudenaarden. 2012. 'Single molecule fluorescent in situ hybridization (smFISH) of C. elegans worms and embryos', WormBook: 1-16.

Jung, C., P. Bandilla, M. von Reutern, M. Schnepf, S. Rieder, U. Unnerstall, and U. Gaul. 2018. 'True equilibrium measurement of transcription factor-DNA binding affinities using automated polarization microscopy', Nat Commun, 9: 1605.

Lanjuin, A., M. K. VanHoven, C. I. Bargmann, J. K. Thompson, and P. Sengupta. 2003. 'Otx/otd homeobox genes specify distinct sensory neuron identities in C. elegans', Dev Cell, 5: 621-33.

Leblond, C. P., and B. E. Walker. 1956. 'Renewal of cell populations', Physiol Rev, 36: 25576.

Leyva-Diaz, E., and O. Hobert. 2019. 'Transcription factor autoregulation is required for acquisition and maintenance of neuronal identity', Development, 146.

Mehta, P., R. Mukhopadhyay, and N. S. Wingreen. 2008. 'Exponential sensitivity of noisedriven switching in genetic networks', Phys Biol, 5: 026005.

Ming, G. L., and H. Song. 2005. 'Adult neurogenesis in the mammalian central nervous system', Annu Rev Neurosci, 28: 223-50.

Ninkovic, J., L. Pinto, S. Petricca, A. Lepier, J. Sun, M. A. Rieger, T. Schroeder, A. Cvekl, J. Favor, and M. Gotz. 2010. 'The transcription factor Pax6 regulates survival of dopaminergic olfactory bulb neurons via crystallin alphaA', Neuron, 68: 682-94.

Orlando, V. 2003. 'Polycomb, epigenomes, and control of cell identity', Cell, 112: 599-606.

Ortiz, C. O., S. Faumont, J. Takayama, H. K. Ahmed, A. D. Goldsmith, R. Pocock, K. E. McCormick, H. Kunimoto, Y. lino, S. Lockery, and O. Hobert. 2009. 'Lateralized gustatory behavior of $\mathrm{C}$. elegans is controlled by specific receptor-type guanylyl cyclases', Curr Biol, 19: 996-1004.

Ozbudak, E. M., M. Thattai, H. N. Lim, B. I. Shraiman, and A. Van Oudenaarden. 2004. 'Multistability in the lactose utilization network of Escherichia coli', Nature, 427: 73740.

Paix, A., A. Folkmann, and G. Seydoux. 2017. 'Precision genome editing using CRISPRCas9 and linear repair templates in C. elegans', Methods, 121-122: 86-93.

Patel, T., and O. Hobert. 2017. 'Coordinated control of terminal differentiation and restriction of cellular plasticity', Elife, 6 .

Raj, A., P. van den Bogaard, S. A. Rifkin, A. van Oudenaarden, and S. Tyagi. 2008. 'Imaging individual mRNA molecules using multiple singly labeled probes', Nat Methods, 5: 877-9.

Ringrose, L., and R. Paro. 2007. 'Polycomb/Trithorax response elements and epigenetic memory of cell identity', Development, 134: 223-32. 
Sarin, S., C. Antonio, B. Tursun, and O. Hobert. 2009. 'The C. elegans Tailless/TLX transcription factor nhr-67 controls neuronal identity and left/right asymmetric fate diversification', Development, 136: 2933-44.

Schindelin, J., I. Arganda-Carreras, E. Frise, V. Kaynig, M. Longair, T. Pietzsch, S. Preibisch, C. Rueden, S. Saalfeld, B. Schmid, J. Y. Tinevez, D. J. White, V. Hartenstein, K. Eliceiri, P. Tomancak, and A. Cardona. 2012. 'Fiji: an open-source platform for biological-image analysis', Nat Methods, 9: 676-82.

Schwanhausser, B., D. Busse, N. Li, G. Dittmar, J. Schuchhardt, J. Wolf, W. Chen, and M. Selbach. 2011. 'Global quantification of mammalian gene expression control', Nature, 473: 337-42.

Sharova, L. V., A. A. Sharov, T. Nedorezov, Y. Piao, N. Shaik, and M. S. Ko. 2009. 'Database for mRNA half-life of 19977 genes obtained by DNA microarray analysis of pluripotent and differentiating mouse embryonic stem cells', DNA Res, 16: 45-58.

Suel, G. M., J. Garcia-Ojalvo, L. M. Liberman, and M. B. Elowitz. 2006. 'An excitable gene regulatory circuit induces transient cellular differentiation', Nature, 440: 545-50.

Teuscher, A. C., and C. Y. Ewald. 2018. 'Overcoming Autofluorescence to Assess GFP Expression During Normal Physiology and Aging in Caenorhabditis elegans', Bio Protoc, 8.

Topalidou, I., and M. Chalfie. 2011. 'Shared gene expression in distinct neurons expressing common selector genes', Proc Natl Acad Sci U S A, 108: 19258-63.

Tursun, B., T. Patel, P. Kratsios, and O. Hobert. 2011. 'Direct conversion of C. elegans germ cells into specific neuron types', Science, 331: 304-8.

Walczak, A. M., J. N. Onuchic, and P. G. Wolynes. 2005. 'Absolute rate theories of epigenetic stability', Proc Natl Acad Sci U S A, 102: 18926-31.

Warren, P. B., and P. R. ten Wolde. 2005. 'Chemical models of genetic toggle switches', J Phys Chem B, 109: 6812-23.

Wicks, S. R., C. J. de Vries, H. G. van Luenen, and R. H. Plasterk. 2000. 'CHE-3, a cytosolic dynein heavy chain, is required for sensory cilia structure and function in Caenorhabditis elegans', Dev Biol, 221: 295-307.

Zhang, L., J. D. Ward, Z. Cheng, and A. F. Dernburg. 2015. 'The auxin-inducible degradation (AID) system enables versatile conditional protein depletion in C. elegans', Development, 142: 4374-84. 